\title{
Combined immunodeficiency due to a mutation in the $\gamma 1$ subunit of the coat protein I complex
}

\author{
Wayne Bainter, ${ }^{1}$ Craig D. Platt, ${ }^{1}$ Seung-Yeol Park, ${ }^{2,3}$ Kelsey Stafstrom, ${ }^{1}$ Jacqueline C. Wallace, ${ }^{1}$ Zachary T. Peters, ${ }^{1}$ \\ Michel J. Massaad, ${ }^{1}$ Michel Becuwe, ${ }^{4}$ Sandra Andrea Salinas, ${ }^{5}$ Jennifer Jones, ${ }^{1}$ Sarah Beaussant-Cohen, ${ }^{1}$ Faris Jaber, ${ }^{1}$ Jia-Shu Yang, ${ }^{2}$ \\ Tobias C. Walther, ${ }^{4}$ Jordan S. Orange, ${ }^{5}$ Chitong Rao, ${ }^{6}$ Seth Rakoff-Nahoum, ${ }^{6}$ Maria Tsokos, ${ }^{7}$ Shafiq Ur Rehman Naseem, ${ }^{8}$ \\ Salem Al-Tamemi, ${ }^{8}$ Janet Chou, ${ }^{1}$ Victor W. Hsu, ${ }^{2}$ and Raif S. Geha ${ }^{1}$
}

'Division of Immunology, Boston Children's Hospital, Harvard Medical School, Boston, Massachusetts, USA. ²Division of Rheumatology, Inflammation and Immunity, Brigham and Women's Hospital, Harvard Medical School, Boston, Massachusetts, USA. ${ }^{3}$ Department of Life Sciences, Pohang University of Science and Technology, Pohang, Gyeongbuk, Republic of Korea. ${ }^{4}$ Department of Cenetics and Complex Diseases and Department of Molecular Metabolism, Harvard T.H. Chan School of Public Health, Boston, Massachusetts, USA. ${ }^{5}$ Division of Immunogenetics, Department of Pediatrics, Morgan Stanley Children's Hospital of New York Presbyterian, Columbia University Irving Medical Center, New York, New York, USA. ${ }^{6}$ Division of Infectious Diseases, Boston Children's Hospital, Harvard Medical School, Boston, Massachusetts, USA. 'Department of Medicine, Beth Israel Deaconess Medical Center, Harvard Medical School, Boston, Massachusetts, USA. ${ }^{8}$ Department of Child Health, Sultan Qaboos University Hospital, Muscat, Oman

The coat protein I (COPI) complex mediates retrograde trafficking from the Golgi to the endoplasmic reticulum (ER). Five siblings with persistent bacterial and viral infections and defective humoral and cellular immunity had a homozygous p.K652E mutation in the $\gamma 1$ subunit of COPI ( $\gamma 1-$ COP). The mutation disrupts COPI binding to the KDEL receptor and impairs the retrieval of KDEL-bearing chaperones from the Golgi to the ER. Homozygous Copg ${ }^{K 652 E}$ mice had increased ER stress in activated T and B cells, poor antibody responses, and normal numbers of T cells that proliferated normally, but underwent increased apoptosis upon activation. Exposure of the mutants to pet store mice caused weight loss, Iymphopenia, and defective T cell proliferation that recapitulated the findings in the patients. The ER stress-relieving agent tauroursodeoxycholic acid corrected the immune defects of the mutants and reversed the phenotype they acquired following exposure to pet store mice. This study establishes the role of $\gamma 1$-COP in the ER retrieval of KDEL-bearing chaperones and thereby the importance of ER homeostasis in adaptive immunity.

\section{Introduction}

During infection, microbial antigens and pathogen-associated molecular pattern molecules activate $\mathrm{B}$ and $\mathrm{T}$ lymphocytes to proliferate and secrete antibodies and cytokines that help eliminate the invading organisms. Secretory proteins, such as immunoglobulins and cytokines, must undergo proper folding in the ER to exit from this compartment for delivery to other intracellular destinations. Several ER-resident chaperones, predominantly immunoglobulin heavy chain binding protein (BiP/GRP-78), calreticulin, and protein disulfide-isomerase (PDI), assist in protein folding and prevent the aggregation of misfolded nascent proteins in the ER (1). These chaperones, which possess a KDEL motif at their carboxyl terminus, do not reside statically in the ER. Instead, a fraction is constantly being leaked to the Golgi complex where they are recognized by the KDEL receptor (KDELR) for retrieval to the ER (2). This retrieval is achieved by the KDELR being transported in vesicles formed by the coat protein I (COPI) complex (3).

Authorship note: WB, CDP, and SYP contributed equally to this work. SAT, JC, VWH, and RSG are equal senior coauthors.

Conflict of interest: The authors have declared that no conflict of interest exists. Copyright: ( 2021, American Society for Clinical Investigation.

Submitted: May 26, 2020; Accepted: November 25, 2020; Published: February 1, 2021

Reference information: J Clin Invest. 2021;131(3):e140494.

https://doi.org/10.1172/JCl140494
COPI performs 2 major functions in mediating retrograde transport: it promotes membrane bending to generate vesicles, and it binds to cargo proteins for their sorting into these vesicles (4). Coatomer, the core component of the COPI complex, is a heptameric complex composed of $\alpha, \beta, \beta^{\prime}, \gamma, \delta$, $\varepsilon$, and $\zeta$ subunits. Different subunits have been identified as recognizing different sequences in cargo proteins (5-8), resulting in a broad range of cargo proteins being packaged into COPI vesicles. A particularly well-characterized mechanism of cargo sorting involves the $\alpha$ and $\beta^{\prime}$ subunits of coatomer recognizing a dilysine motif in cargo proteins. Structural studies revealed that the binding pocket of $\alpha / \beta^{\prime}-$ COP can only accommodate the dilysine residues when they are located near the carboxyl terminus of cargo proteins, specifically $\operatorname{KKXX}$ or $\operatorname{KXKXX}(9,10)$. The $\operatorname{KDELR}$ possesses dilysine residues in its cytoplasmic carboxyl tail. However, the dilysine residues are located further away from the carboxyl terminus (KKXXXXX), which is predicted to prevent binding by $\alpha / \beta^{\prime}-\mathrm{COP}(9,10)$. Thus, how coatomer binds the KDELR for retrograde transport from the Golgi to the ER remains to be determined.

In the ER, an increase in the load of nascent unfolded proteins, or the accumulation of mutant misfolded proteins, leads to ER stress and triggers the unfolded protein response (UPR) (11). The UPR is the mechanism responsible for expanding the secretory capacity of the cell, thereby alleviating ER stress. The elevated levels of misfolded proteins in the ER cause the chaperone 


\section{Table 1. Phenotypic and functional evaluation of blood lymphocytes}

\begin{tabular}{|c|c|c|c|c|c|}
\hline & P1 & P2 & P3 & P4 & P5 \\
\hline Age at evaluation & 12 years & 9 years & 8 years & 2.5 years & 9 months \\
\hline \multicolumn{6}{|c|}{ Lymphocyte subsets (cells/ $\mu \mathrm{L}$ ) } \\
\hline $\mathrm{CD}^{+}$ & $974(1000-2200)$ & $379(1200-2600)$ & $438(1200-2600)$ & $550(1400-3700)$ & $1620(1900-5900)$ \\
\hline $\mathrm{CD}^{+} \mathrm{CD}^{+}$ & $824(330-920)$ & $268(370-1100)$ & $260(370-1100)$ & $456(490-1300)$ & $988(500-1700)$ \\
\hline $\mathrm{CD}^{2} 5 \mathrm{RA}^{+}(\%)$ & 70 (39-93) & 85 (47-97) & 47 (47-97) & $90(53-96)$ & $83(68-98)$ \\
\hline $\mathrm{CD}^{2} 5 \mathrm{RO}+(\%)$ & $30(18-68)$ & $15(8-76)$ & $53(8-76)$ & $10(7-50)$ & $16(4-29)$ \\
\hline $\mathrm{CD}_{16} \mathrm{CD}^{\mathrm{C}} \mathrm{6}^{+}$ & $168(70-480)$ & $86(100-480)$ & $39(100-480)$ & $2(130-720)$ & $181(160-950)$ \\
\hline$\gamma \delta \mathrm{T}$ cells & $369(124-388)$ & $194(124-388)$ & $171(124-388)$ & nd & $196(128-436)$ \\
\hline \multicolumn{6}{|c|}{ T cell proliferation, ${ }^{3} \mathrm{H}-\mathrm{Td} \mathrm{cpm}$} \\
\hline Medium & $134(270)$ & $301(270)$ & $120(270)$ & $130(270)$ & nd \\
\hline PHA & $1149(119,452)$ & $7043(119,452)$ & $169(119,452)$ & $4798(119,452)$ & nd \\
\hline Anti-CD3 & $257(42,901)$ & $2055(42,901)$ & $118(42,901)$ & $2746(42,901)$ & nd \\
\hline
\end{tabular}

Abnormal values are indicated in bold. Values of T, B, and NK cell markers shown in parentheses represent the normal range for age. Values of T cell proliferation and B cell IgG secretion shown between parentheses represent the mean of 2 healthy controls whose blood was shipped together with the patients' blood. nd, not done; ${ }^{3} \mathrm{H}-\mathrm{Td},\left[{ }^{3} \mathrm{H}\right]$-thymidine.

BiP to dissociate from 3 ER stress sensors to which it is normally bound: inositol requiring kinase-1 $\alpha$ (IRE1), PKR-like ER-related kinase (PERK), and activated transcription factor 6 (ATF6) (12, 13). Upon dissociation of BiP, IRE1 $\alpha$ and PERK dimerize and transautophosphorylate, thereby activating their kinase domain, while ATF6 translocates to the Golgi and is activated upon cleavage by the proteases S1P and S2P $(14,15)$. Each arm of the UPR offers complementary mechanisms to alleviate ER stress and effectively increase the protein-folding capacity of the ER (11). Activated $\mathrm{B}$ and $\mathrm{T}$ cells are prone to ER stress because of their high rate of protein secretion, but normally the UPR is sufficient to support the increased secretory load.

The IRE1 $\alpha$ arm of the UPR has been widely viewed as the most essential. Once activated, the endoribonuclease domain of IRE1 $\alpha$ specifically splices a 26-nucleotide intronic sequence from inactive Xbp1 mRNA, a downstream product of ATF6, which creates a stable active form known as spliced XBP1 (sXBP1) (16). sXBP1 migrates to the nucleus, where it acts as a transcription factor that upregulates the expression of many genes involved in the secretory pathway, membrane biogenesis, ER chaperones, protein synthesis, and protein degradation via ER-associated degradation (ERAD) (17). sXBP1 has been shown to be essential for the differentiation and function of antibody-secreting plasma cells $(17,18)$.

While the IRE1 pathway leads to molecular changes that allow the ER to handle the increased protein load, the PERK arm of the UPR aims to reduce ER stress by another mechanism. Once phosphorylated, phosphorylated PERK (p-PERK) recruits and phosphorylates eIF $2 \alpha$, which inhibits global protein translation, thereby reducing the protein load in the ER. One of the few mRNAs excluded from eIF $2 \alpha$ inhibition is the mRNA encoding ATF4, a transcription factor that drives the expression of Ddit3, which encodes the CCAAT enhancer-binding protein homologous protein (CHOP)
(19). Under chronic ER stress, CHOP drives the expression of the proapoptotic factors Bim, GADD34, and DR5 while simultaneously downregulating the expression of the antiapoptotic factor BCL-2. This ultimately leads to apoptosis of overstressed cells (20, 21). Ddit3 expression is upregulated in ER-stressed T cells, but is downregulated in activated B cells (22-24). CD4 ${ }^{+} \mathrm{T}$ cells are more susceptible than $\mathrm{CD}^{+}{ }^{+} \mathrm{T}$ cells to $\mathrm{CHOP}$-driven cell death because they express lower levels of the antiapoptotic protein BCL-2 (25).

Combined immunodeficiency (CID) is a primary immunodeficiency characterized by impaired humoral and cell-mediated immunity, leading to susceptibility to life-threatening infections. The genetic basis of many cases of CID remains unknown. We have identified a cause of CID due to a homozygous missense mutation in the $\gamma 1$ subunit of COPI ( $\gamma 1-\mathrm{COP})$. We demonstrate that the mutation disrupts the binding of the KDELR by coatomer, which results in defective retrieval of ER chaperones, leading to increased ER stress that underlies the patients' defective humoral and cellular immunity.

\section{Results}

Patient characteristics. Five Omani siblings, born to consanguineous parents, presented in the first year of life with recurrent respiratory infections. They all suffered from recurrent pneumonias and failure to thrive (weight and height below third centile). $H$. influenzae, S. pneumoniae, and Moraxella were cultured from their sputum (Supplemental Table 1; supplemental material available online with this article; https://doi.org/10.1172/JCI140494DS1). Viruses, including influenza A, adenovirus, respiratory syncytial virus, rhinovirus, and parainfluenza 3 , were detected by immunofluorescence in the patients' sputum during acute respiratory infections (Supplemental Table 1). Bronchiectasis was present in patients 1, 2, and 3 (P1-P3) (Supplemental Table 1). All 5 patients had hepatitis, and all suffered from persistent CMV viremia (Sup- 
A

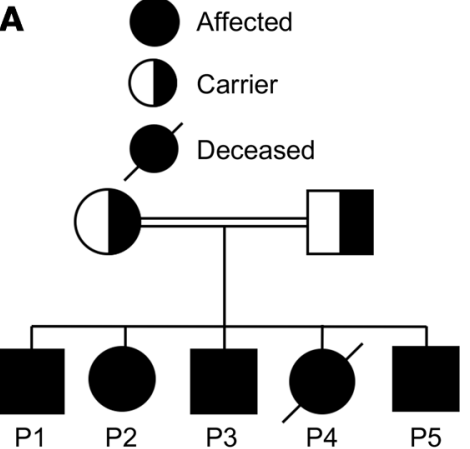

B

Control

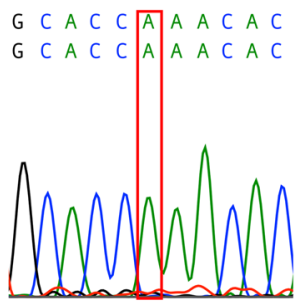

Mother

$G C A C C A A A C A C$
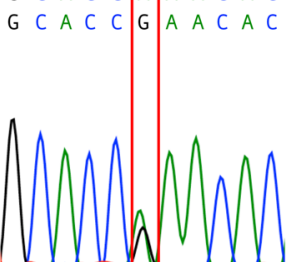

Patient

$G C A C C G A A C A C$ $G C A C C G A A C A C$

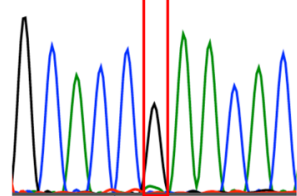

C

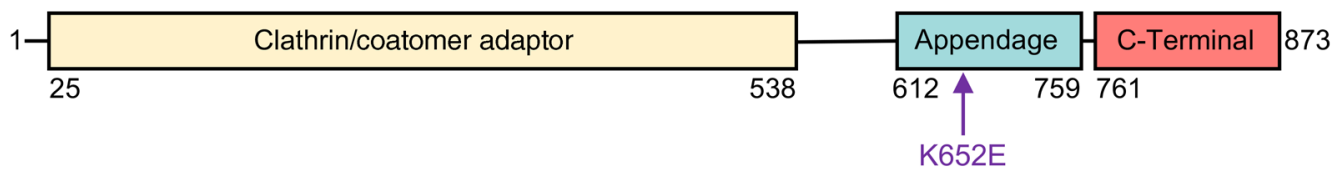

D

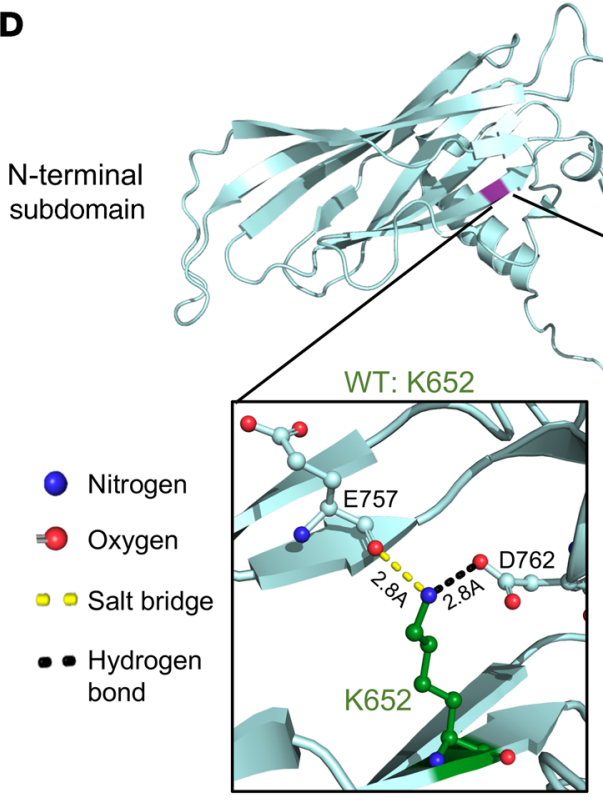

$\mathbf{F}$



C-terminal subdomain

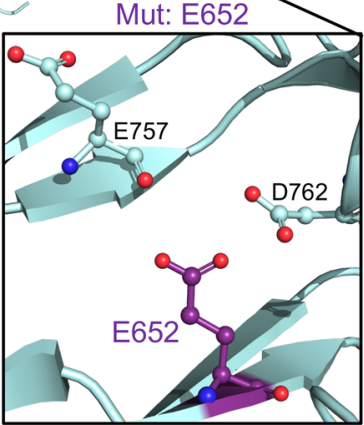

$\mathbf{E}$
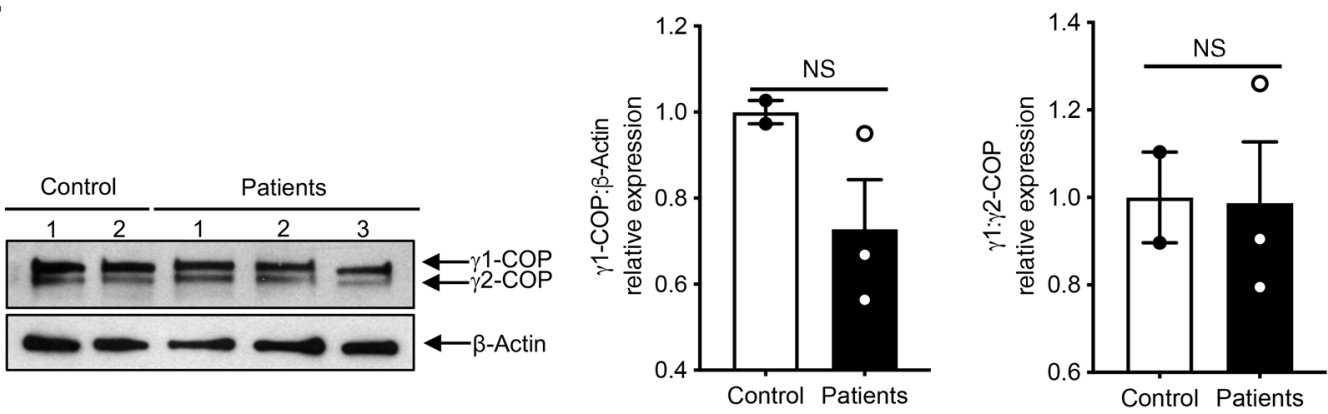

Figure 1. Homozygous COPG ${ }^{1652 E}$ mutation in the patients. (A) Family pedigree. (B) Sanger sequencing of the region surrounding the mutation in COPG1 (c.A1954G:p.K652E) in control, mother, and P1. (C) Linear map of $\gamma 1$-COP. Arrow indicates the location of the K652E missense mutation in the appendage domain. (D) Ribbon diagram of the human $\gamma 1$-COP appendage domain (aa 608-874) and its $\mathrm{N}$-terminal and C-terminal subdomains. Insets show the potential impact of the mutation on the local interactions between the WT K652 (left) or mutant E652 residue (right) and residues E757 in the N-terminal subdomain, and D762 in the C-terminal subdomain. (E) Representative immunoblot of $\gamma$-COP ( $\gamma 1-\operatorname{COP}$ and $\gamma 2$-COP) in fibroblasts from P1, P2, and P3 ( $n=3)$ and controls $(n=2)$ (top) and quantitation of $\gamma 1-\mathrm{COP} / \beta$-actin and $\gamma 1-\mathrm{COP} / \gamma 2$-COP ratios in patients relative to controls in 2 independent experiments (bottom). (F) Representative $\gamma$-COP ( $\gamma 1-\mathrm{COP}$ and $\gamma 2-\mathrm{COP})$ and $\beta$-COP immunoblot of lysates and of $\beta$-COP and IgG control immunoprecipitates, from P2 and control fibroblasts (top) and quantitation of $\gamma 1$-COP/ $\beta$-COP ratio in patients relative to controls ( $n=2$ each) in 3 independent experiments (bottom). Columns and bars represent mean \pm SEM. 
plemental Table 1). Two of the patients (P1 and P4) had chronic EBV viremia, while $\mathrm{P} 4$ had persistent parvovirus B19 viremia (Supplemental Table 1). P5 received a genetic diagnosis at birth. By 6 months of age, he presented with a chest infection and was found to have CMV viremia (Supplemental Table 1). Given their chronic bacterial and viral infections, the patients received the diagnosis of CID and were started on gammaglobulin replacement shortly after presentation. $\mathrm{P} 4$ died at age 3.5 years with respiratory failure and multiorgan dysfunction.

At presentation, serum IgG levels were elevated in P2 and P4 and normal in P1 and P5 (Supplemental Table 2). Serum IgA and IgM levels were normal, or near normal, in all 4 patients. Serum immunoglobulins were not available for P3. Antibody responses were available for P1 and P2; they showed poor response to tetanus and diphtheria booster vaccine $(<4$-fold rise in titer) and absent antibody response to hepatitis B and measles vaccination. Autoantibodies were not detected in the patients' sera using the University of Texas Southwestern autoantigen microarray panel of 128 human autoantigens. P1-P5 had severe CD4 ${ }^{+} \mathrm{T}$ cell lymphopenia, with variable and modest $\mathrm{CD}^{+} \mathrm{T}$ cell lymphopenia both at presentation (Supplemental Table 2) and later on at the time of evaluation of in vitro immune function (Table 1). Notably, P5 had normal numbers of CD4 $4^{+} \mathrm{T}$ cells $(1591$ cells/ $\mu \mathrm{L})$ and $\mathrm{CD} 8^{+} \mathrm{T}$ cells $(1051$ cells $/ \mu \mathrm{L})$ in cord blood. The percentages of naive and memory $\mathrm{CD}^{+}$and $\mathrm{CD} 8^{+} \mathrm{T}$ cells, as well as the numbers of $\gamma \delta \mathrm{T}$ cells were comparable in the patients and controls (Table 1). B cell and NK cell numbers were normal or variably diminished in the patients (Supplemental Tables 1 and 2). T cell proliferation in response to stimulation with phytohemagglutinin (PHA) and anti$\mathrm{CD} 3 \mathrm{mAb}$ was markedly reduced in all 4 patients studied (P1-P4) compared with healthy shipping controls. In vitro secretion of IgG in response to stimulation with anti-CD40+IL-4 was also diminished in the 4 patients studied (P1-P3 and P5) compared with controls (Table 1).

Homozygous COPG1 ${ }^{K 652 E}$ mutation in the patients. Whole-exome sequencing (WES) was performed on 3 patients and their mother. Six nonsynonymous homozygous mutations were identified in the 3 patients and were heterozygous in the mother; all were absent from the 1000 Genomes and ExAC databases. Autozygosity mapping of the patients revealed a shared region of homozygosity on chromosome 3 (126611300-134019500). A single variant was identified within this region, COPG1 (c.A1954G: p.K652E), which encodes the $\gamma 1$-COP subunit of the COPI coatomer. Sanger sequencing revealed that the COPG ${ }^{K 652 E}$ mutation was homozygous in the 5 affected siblings and heterozygous in both parents (Figure 1, A and B).

The $\mathrm{K} 652$ residue is located within the appendage domain of $\gamma 1$-COP (Figure 1C) and is highly conserved (Supplemental Figure 1). The crystal structure of $\gamma 1$-COP revealed that K652 is located at the $\mathrm{N}$-terminal end of the appendage domain (26). Structural modeling predicts that the K652E mutation may disrupt 2 potential bonds that could alter the orientation of the 2 appendage subdomains of 1 1-COP: a salt bridge between K652 and the carboxyl side chain of E757 and a hydrogen bond between K652 and the main chain carbonyl oxygen of D762 (Figure 1D). Immunoblotting with an antibody that detects $\gamma 1$-COP and its paralog $\gamma 2$-COP revealed comparable expression of $\gamma 1-\mathrm{COP}$ in fibroblasts from patients and controls (Figure 1E). Coprecipitation of $\gamma 1-\mathrm{COP}$ with the $\beta$-COP subunit of COPI was comparable in patients and controls (Figure $1 \mathrm{~F}$ ), suggesting that the mutant $\gamma 1$-COP integrates normally into the COPI complex.

The COPG1 ${ }^{\text {K652E }}$ mutation impairs retrograde protein trafficking of VSVG-KDELR fusion protein and disrupts the association of $\gamma 1$-COP with KDELR. We next pursued studies of COPI function. An assay has been established to track retrograde COPI transport in cells (27-29). Briefly, it involves fusing the lumenal portion of a temperature-sensitive mutant of the vesicular stomatitis virus $\mathrm{G}$ protein $\left(\mathrm{VSVG}^{\mathrm{ts}}\right.$ ) to the KDELR, a cargo protein of retrograde COPI transport that cycles between the ER and the Golgi (2, 3, 30). At the permissive temperature, the VSVG ${ }^{\text {ts }}-K D E L R$ fusion protein shuttles between the ER and the Golgi apparatus and has a steady-state distribution mostly at the Golgi. Upon shift to the nonpermissive temperature, anterograde transport is selectively blocked, allowing 1 round of retrograde COPI transport to be tracked unambiguously (refs. 27-29; also summarized in Figure 2, A and B). Performing this transport assay, we found that retrograde COPI transport in skin-derived fibroblasts of affected patients was impaired, as reflected by increased colocalization of the VSVG-KDELR with GM130, a marker of the cis-Golgi, in the mutant cells (Figure 2C). This result was also confirmed by quantitation (Figure $2 \mathrm{C}$ ).

To further determine whether the $\gamma 1$-COP mutation causes defective vesicular transport, we performed CRIPSR/Cas9 genome editing to generate mice that expressed the homozygous Copg1 ${ }^{\text {K652E }}$ mutation. Embryonic fibroblasts derived from these mice (MEFs) expressed the mutant $\gamma 1$-COP at levels similar to those of WT $\gamma 1-\mathrm{COP}$ in control MEFs (Figure 2D). We found that mutant MEFs also exhibited defective retrograde COPI transport (Figure 2E), similar to that seen in cells derived from affected patients.

To elucidate the mechanistic basis of this defect, we examined the 2 major functions of COPI in transport, promoting vesicle formation and binding to cargo proteins (4). Coatomer was purified from livers of both WT and mutant mice, which again confirmed that the $\gamma 1$-COP mutation did not affect the assembly of coatomer subunits into a heptameric complex (Supplemental Figure 2A). To examine vesicle formation, we performed the reconstitution of COPI vesicles from Golgi membrane as previously described (refs. 28, 31; also summarized in Supplemental Figure 2B). In brief, Golgi membrane was incubated with purified ADP-ribosylation factor 1 (ARF1) and coatomer to initiate COPI assembly on the membrane. The coated membrane was then reisolated by centrifugation and incubated with recombinant forms of a GTPase-activating protein (GAP) for ARF1, known as ARFGAP1, and brefeldin-A ADP-ribosylated substrate (BARS) to drive COPI vesicle formation to completion. Performing this reconstitution assay, we found that the mutant coatomer promoted COPI vesicle formation similarly to WT coatomer (Supplemental Figure 2, C and D), indicating that the $\gamma 1$-COP mutation had no appreciable effect on the role of coatomer in vesicle formation.

We then examined cargo binding by coatomer. To assess this function, we had previously fused the cytoplasmic tail of the KDELR to glutathione-S-transferase (GST), and then gathered the fusion protein onto beads for incubation with purified coatomer $(28,31)$. Performing this binding assay, we found that the $\gamma 1$-COP 
A

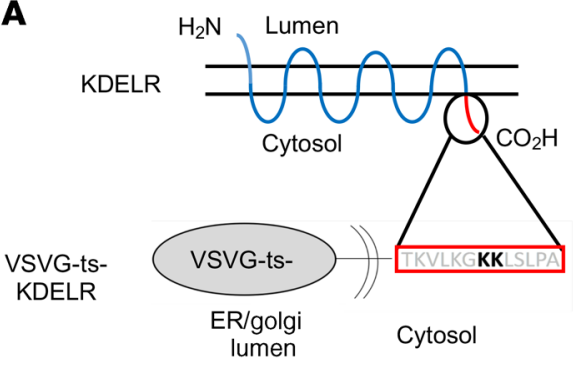

B

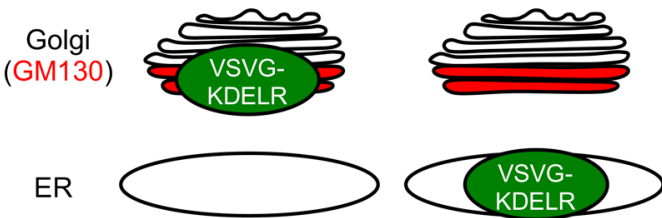

C
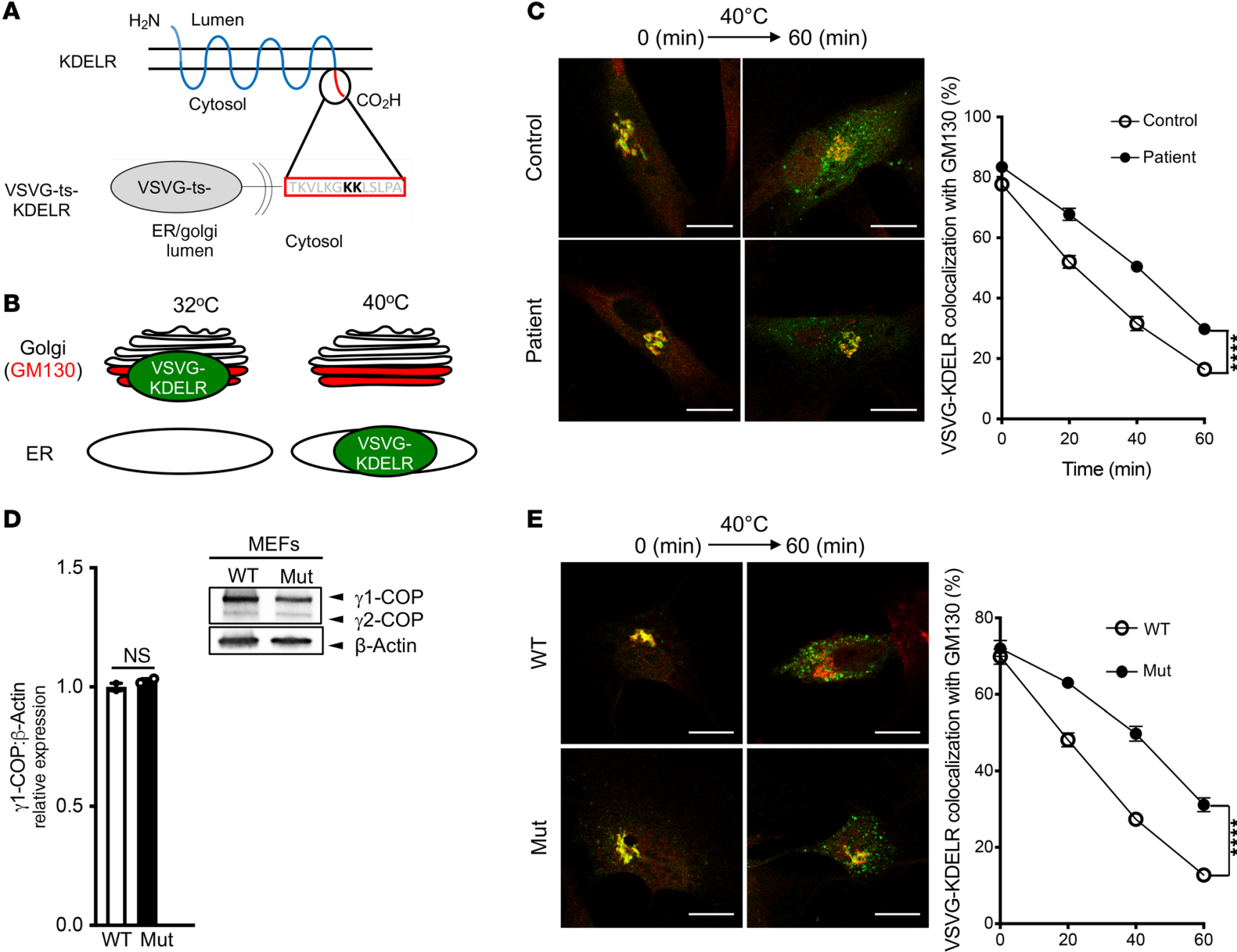

MEFs

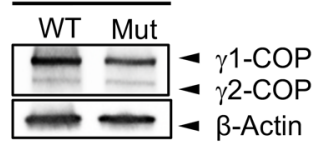

$\mathbf{F}$

$\underline{\text { WT COPI Mut COPI }}$

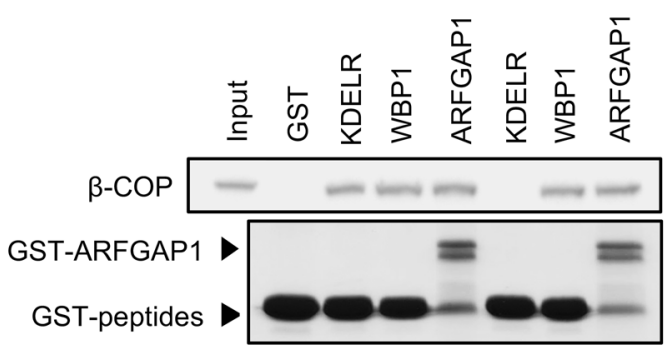

E
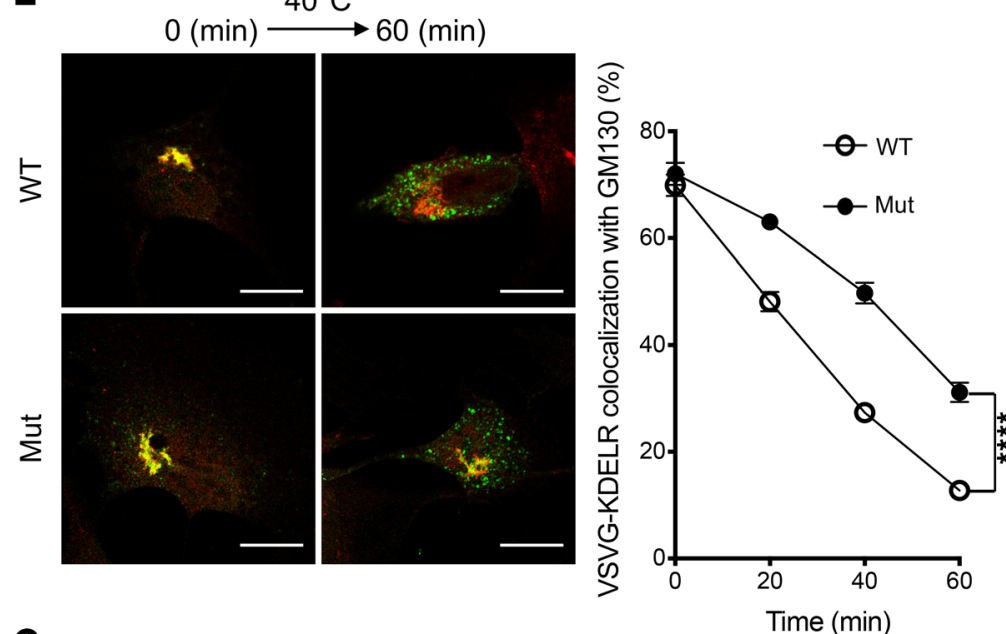

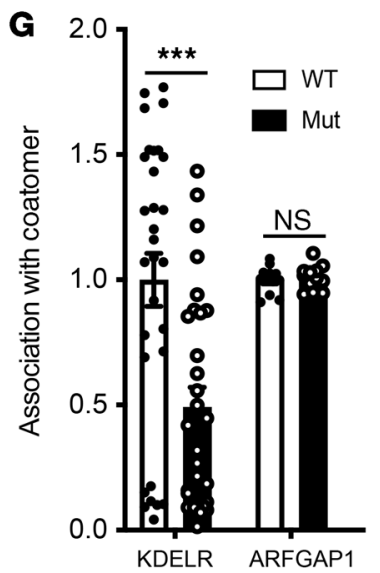

Figure 2. The COPG ${ }^{K 652 E}$ mutation impairs the transport of a retrograde COPI-dependent cargo and its association with coatomer. (A and B) Experimental schematic. (C) Representative confocal images (left) showing the colocalization of VSVG-KDELR (green) and a Golgi marker (GM130, red) in control and patient fibroblasts 60 minutes after shifting to the nonpermissive temperature. Scale bars: $5 \mu \mathrm{M}$. Quantitation of this colocalization (right); $n=10$ cells examined per time point per sample. (D) Immunoblotting of whole cell lysates assessing the level of $\gamma$-COP isoforms ( $\gamma 1$-COP and $\gamma 2$-COP) in MEFs from Copg ${ }^{K 652 E}$ and WT mice (top) and quantitation of the $\gamma 1$-COP level relative to $\beta$-actin (bottom). $n=2$ experiments. (E) Copg ${ }^{K 552 E}$ MEFs show defective retrograde COPI transport. Representative confocal images (left) showing the colocalization of VSVG-KDELR (green) and a Golgi marker (GM130, red) in MEFs from WT and mutant mice 60 minutes after shifting to the nonpermissive temperature. Scale bars: $5 \mu \mathrm{M}$. Quantitation of the colocalization (right). $n=10$ cells examined per time point per sample. (F) Copg ${ }^{K 652 E}$ impairs the direct binding of coatomer to the KDELR cytoplasmic tail. Pulldown study performed by incubating GST fused to different proteins (as indicated) on beads with soluble coatomer (WT or mutant), followed by immunoblotting of the beads for $\beta$-COP (top) and GST (below). (C) Copg ${ }^{\text {K652E }}$ impairs the association of the KDELR with coatomer in cells. FLIM was performed in MEFs to examine the association of coatomer with either KDELR or ARFCAP1. Results are expressed relative to the mean of WT control ( $n=30$ for KDELR and $n=12$ for ARFGAP1). A representative result from 3 experiments is shown in C, E, F, and $\mathbf{G}$ Quantitation in (C, D, E, and $\mathbf{G})$ represents mean \pm SEM. ${ }^{* *} P<0.001 ;{ }^{* * *} P \leq 0.0001$, 2-tailed Student's $t$ test (D and G); 2 -way ANOVA (C and E). 

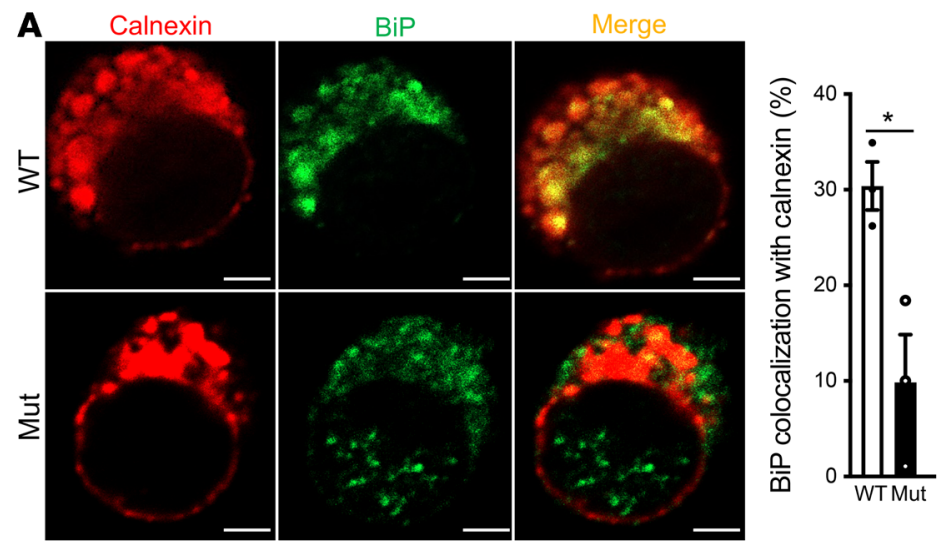

E
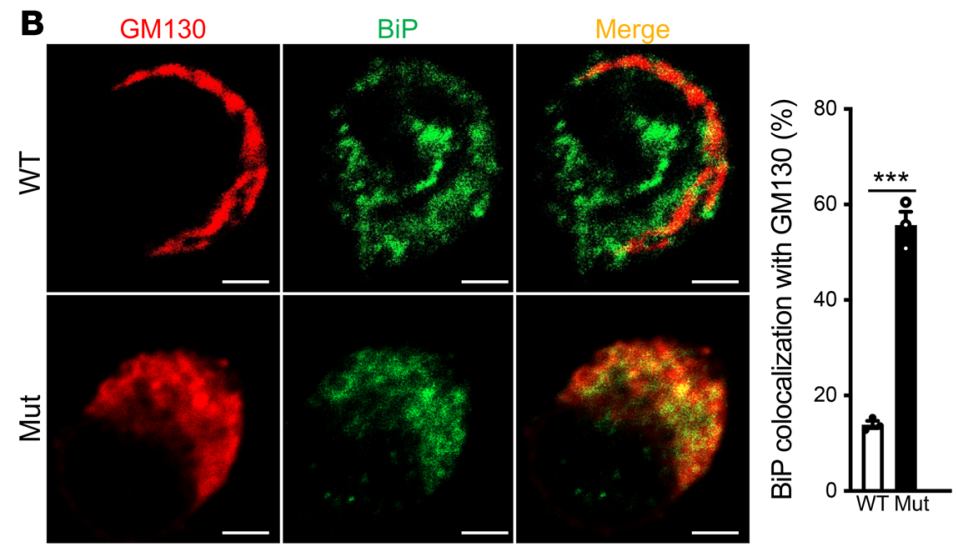

C


$\mathbf{F}$
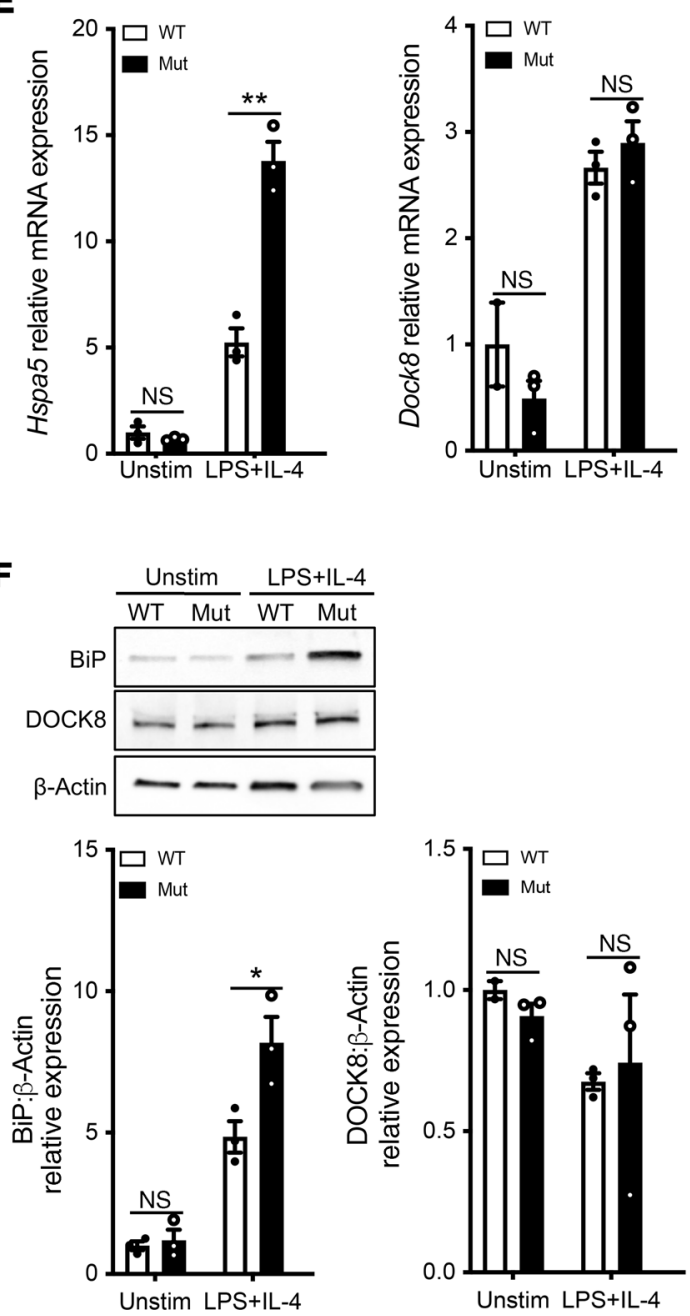

G
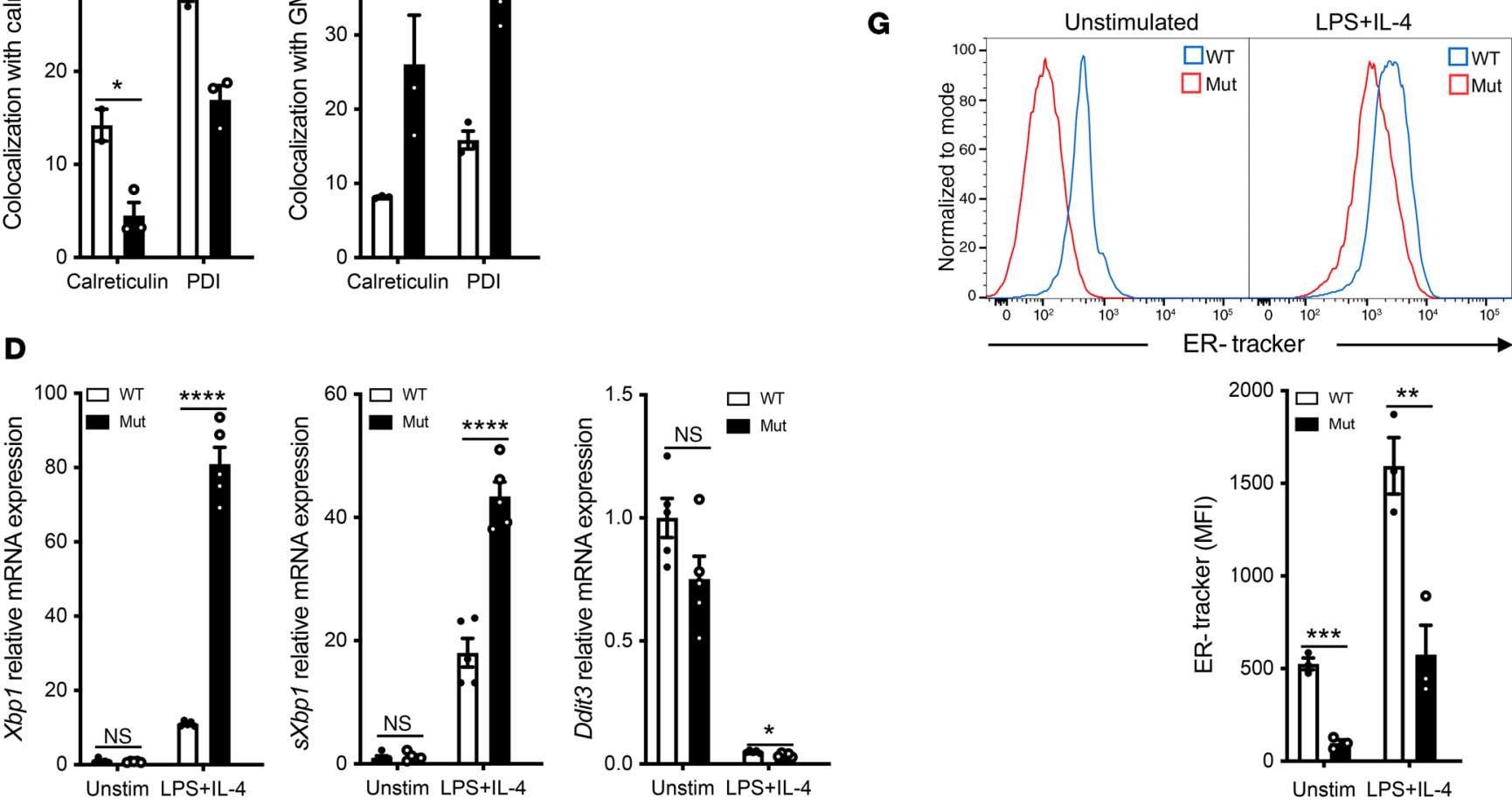
Figure 3. Activated B cells from homozygous Copg $1^{\text {K652E }}$ mutant mice have mislocalized BiP and increased ER stress. (A) Representative images of LPS+IL-4 stimulated B cells from mutant mice and controls stained for the ER marker calnexin (red) and BiP (green) and merged (yellow) (left). Scale bars: $2 \mu \mathrm{M}$. Quantitation of colocalization of calnexin and BiP (right) with a representative result from 3 independent experiments shown. $n=3$ cells examined in each. (B) Representative images of $B$ cells from the same experiment in A, stained for the Golgi marker GM130 (red) and BiP (green) and merged (yellow) (left). Scale bars: $2 \mu \mathrm{M}$. Quantitation of colocalization of $\mathrm{CM} 130$ and $\mathrm{BiP}$ (right) with a representative result from 3 independent experiments shown. $n=3$ cells examined in each. (C) Quantitation of colocalization of calreticulin and PDI with calnexin and with GM130 in LPS+IL-4 stimulated $B$ cells from mutant mice and WT controls. (D) Relative mRNA expression of the UPR response genes $X b p 1, s X b p 1$, and Ddit3 in unstimulated and LPS+IL-4 stimulated B cells from mutant mice and WT controls. $n=3$ independent experiments each with 5 mice per group. (E) Relative Hspa 5 and Dock 8 mRNA expression. $n=3$ independent experiments each with 3 mice per group. (F) Representative immunoblot of BiP and DOCK8 in unstimulated and LPS+IL-4 stimulated B cells from mutant mice and WT controls and quantitative analysis of the results of BiP or DOCK8 expression relative to WT control from 3 experiments with 3 mice per group. (C) Representative histogram analysis of ER-Tracker dye uptake by unstimulated and LPS+IL-4 stimulated mutant and WT B cells (left) and quantitation of MFI of ER-Tracker dye in 4 experiments each with 3 mice/ group (right). Columns and bars represent mean \pm SEM. ${ }^{*} P \leq 0.05$; ${ }^{* *} P \leq$ $0.01 ;{ }^{* *} P \leq 0.001 ;{ }^{* * *} P \leq 0.0001,2$-tailed Student's $t$ test.

mutation disrupted the direct binding of coatomer to the KDELR tail (Figure 2F). As control, the $\gamma 1-\mathrm{COP}$ mutation did not affect the direct binding of coatomer to Wbp1 (Figure 2F), a COPI cargo protein that possesses a classic dilysine motif (KKXX) and is predicted to bind coatomer through the $\alpha / \beta^{\prime}$ subunits $(9,10,32)$.

We also addressed a seeming disparity. The $\gamma 1$-COP mutation disrupted the direct binding of coatomer to the KDELR (Figure $2 \mathrm{~F}$ ), but had a more modest effect in reducing the retrograde transport of VSVG-KDELR (Figure 2, C and E). ARFGAP1 has been shown to act as another component of the COPI complex by linking coatomer to cargoes including KDELR (33). We found that the $\gamma 1$-COP mutation had no appreciable effect on coatomer binding to ARFGAP1 (Figure 2F). Furthermore, fluorescence lifetime imaging microscopy (FLIM) revealed that coatomer with the $\gamma 1$-COP mutation showed partial reduction in interacting with the KDELR, but retained full interaction with ARFGAP1 (Figure $2 \mathrm{G}$ ). Thus, disruption of the direct interaction between the mutant coatomer and the KDELR may be partially compensated for by indirect interaction of the mutant coatomer with the KDELR through ARFGAP1.

Normal lymphocyte development in homozygous Copg1 $1^{\mathrm{K} 652 \mathrm{E}}$ mutant mice. We then examined mice that expressed the mutant $\gamma 1$-COP to gain insight into how the impaired transport of the KDELR from the Golgi to the ER could explain the immunologic defects observed in patients. We observed that the bone marrow of Copg1 $1^{K 652 E}$ mice had normal cellularity and B cell development (Supplemental Figure 3A). Their thymus, spleen, and lymph nodes (LNs) had normal cellularity (Supplemental Figure 3B). Thymocyte development was normal (Supplemental Figure 3C). Mutant mice had normal numbers of splenic $\mathrm{CD}^{+}$cells, B220 ${ }^{+} \mathrm{B}$ cells, $\gamma \delta$ T cells, MAIT cells, iNKT cells, innate lymphoid cells (ILCs), and CD3-NK1. $1^{+}$NK cells (Supplemental Figure 3D). Mutant T cells and $\mathrm{B}$ cells had $\gamma 1$-COP expression comparable to that of WT con- trols (Supplemental Figure 3E). The distribution of splenic B cell subsets, including $\mathrm{T} 1\left(\mathrm{CD} 93^{+} \mathrm{CD} 23^{-} \operatorname{IgM}^{+}\right)$, T2 $\left(\mathrm{CD}^{+} 3^{+} \mathrm{CD} 23^{+} \mathrm{IgM}^{+}\right)$, follicular cells $(\mathrm{FO})\left(\mathrm{CD} 93^{-} \mathrm{CD} 23^{+} \mathrm{CD} 21^{\text {int }}\right)$, and marginal zone (MZ) $\left(\mathrm{CD} 93^{-} \mathrm{CD} 23^{+} \mathrm{CD} 21^{+}\right) \mathrm{B}$ cells, was normal in the mutant (Supplemental Figure 3F). The distribution of $\mathrm{CD}^{+}$and $\mathrm{CD}^{+}$ $\mathrm{T}$ cells in spleens, inguinal LNs, and mesenteric LNs (MLNs) as well as the percentages of splenic naive and memory $\mathrm{CD} 4^{+}$cells and $\mathrm{CD}^{+}$cells, and of $\mathrm{CD} 4^{+} \mathrm{FOXP}^{+}$Tregs and $\mathrm{CD}^{+}{ }^{+} \mathrm{FOXP3}^{+} \mathrm{C}-$ $\mathrm{CXR5}^{+}$follicular Tregs were comparable in the mutants and WT controls (Supplemental Figure 3G). Furthermore, the histology of the spleen was comparable in the mutants and WT controls (Supplemental Figure $3 \mathrm{H})$. Moreover, surface expression of TCR- $\beta$, CD3e, CD4, and CD8 on T cells, B220 and CD19 on B cells, and the TLR4/MD2 complex on peritoneal macrophages was comparable in mutants and WT controls (Supplemental Figure 3, I and J). Autoantibodies were not detected in the sera of mutant mice using the University of Texas Southwestern Microarray Super Panel of 128 mouse autoantigens. These results indicate that the $\gamma 1$-COP mutation does not interfere with the development of T and B cells.

Activated B cells from Copg1 mutant mice have mislocalized ER chaperones and increased ER stress. A prediction of reduced retrieval of the KDELR in mutant cells is that the retrieval of KDEL-bearing ER chaperones would also be impaired. Confocal microscopy revealed significantly less colocalization of the KDEL-bearing chaperone BIP with calnexin, an ER marker, in LPS+IL-4 activated mutant B cells as compared with similarly treated WT cells (Figure 3A). Moreover, there was greater colocalization of BIP with GM130, a Golgi marker, in mutant B cells compared with that seen in WT cells (Figure 3B). Similar to BIP, the KDEL-bearing chaperones calreticulin and PDI were mislocalized in LPS+IL-4 activated mutant $\mathrm{B}$ cells, with less in the ER and more in the Golgi (Figure 3C). These results confirmed that the Copg $1^{\text {K652E }}$ mutation also results in the defective retrieval of KDEL-bearing chaperones from the Golgi to the ER.

The increased load of unfolded immunoglobulins in the ER of activated normal B cells has been shown to cause ER stress (18). Thus, defective retrieval of ER chaperones would be expected to induce exaggerated ER stress and increased activation of the UPR in the activated mutant B cells. We found that the expression of $X b p 1$ and $s X b p 1$ was significantly higher in LPS+IL-4 activated mutant B cells than in WT B cells (Figure 3D). In contrast, the expression of Ddit3, decreased in activated WT and mutant B cells (Figure 3D), consistent with the known downregulation of Ddit3 expression during B cell differentiation into plasmablasts $(23,24)$. XBP1 drives expression of Hspa5, which encodes BiP (34). Expression of Hspa5 mRNA and BIP was significantly higher in LPS+IL-4 activated mutant B cells compared with WT B cells (Figure 3, E and F). Dock8 mRNA and DOCK8 expression were comparable, indicating that the mutant $\mathrm{B}$ cells do not have a global alteration in mRNA and protein expression. B cell activation is associated with an increase in ER size (35). ER size was examined by flow cytometry analysis of the uptake of the ER selective dye, ER-Tracker Green. ER size was significantly decreased in resting and LPS+IL-4 activated mutant B cells compared with WT controls (Figure $3 G$ ), suggesting that impaired retrieval of ER chaperones adversely affected ER integrity in the mutant $\mathrm{B}$ cells. Electron microscopic examination revealed 
A

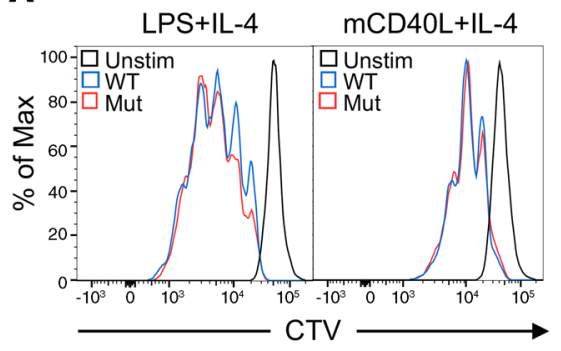

D

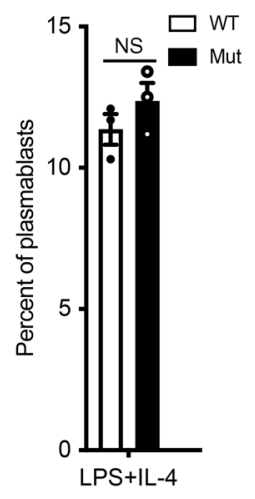

H

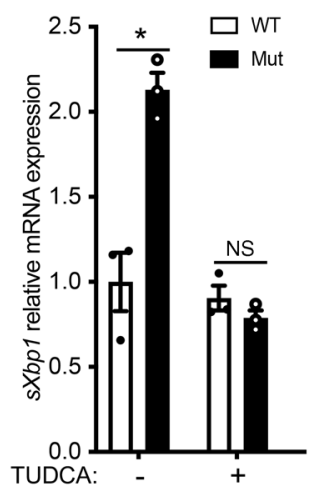

E
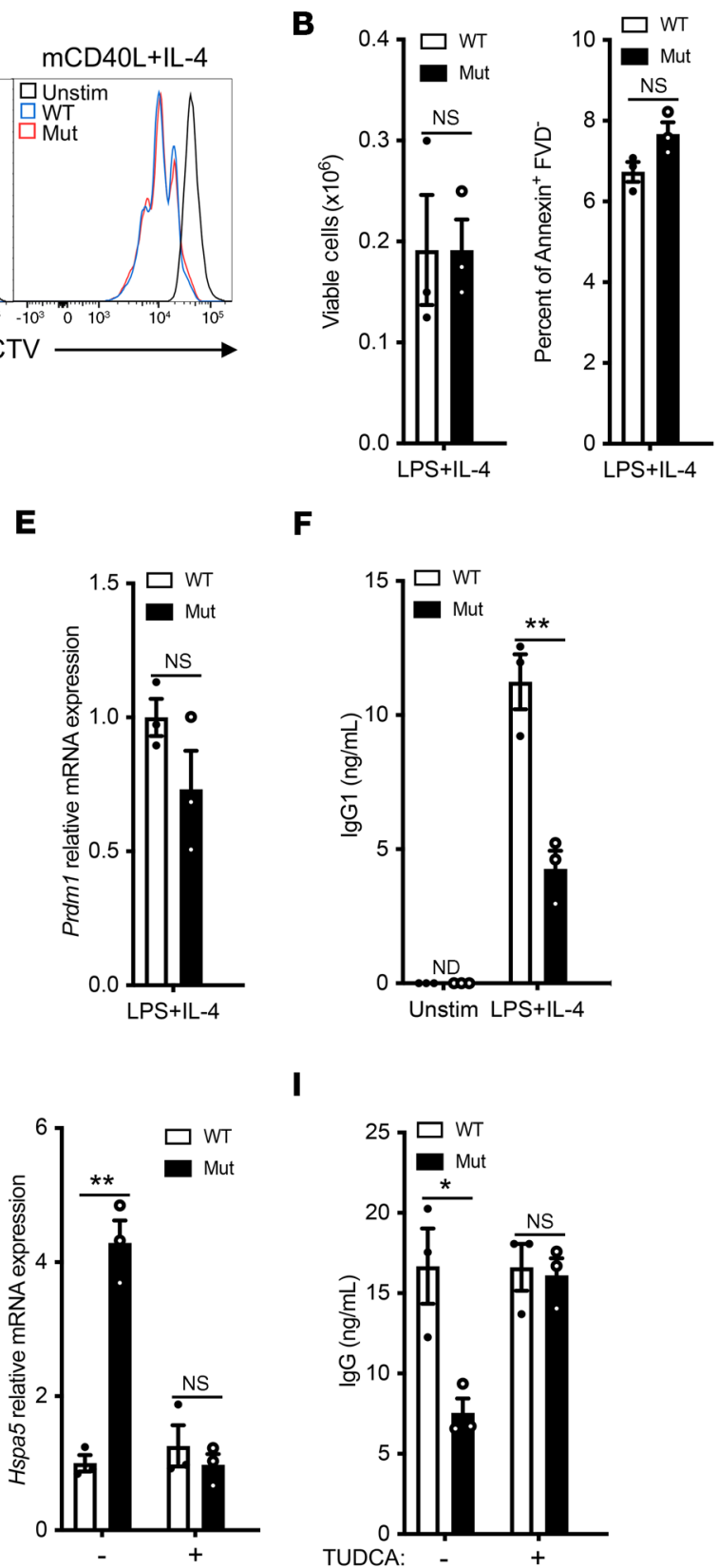

$\mathbf{K}$

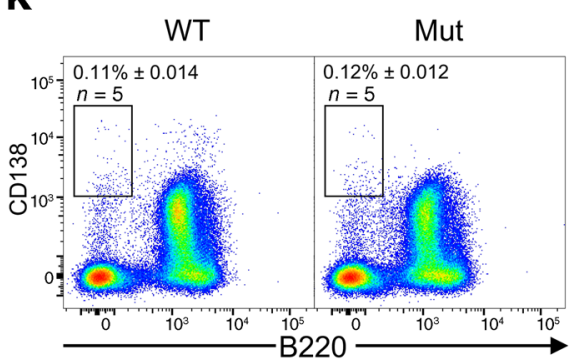

$\mathbf{F}$
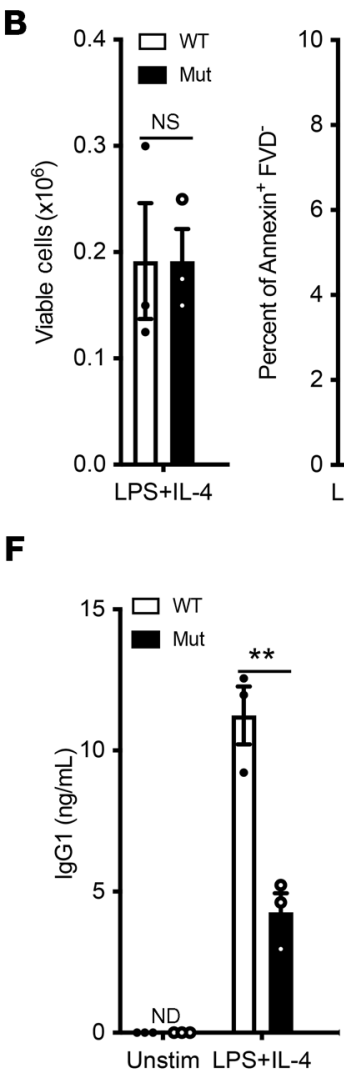

I

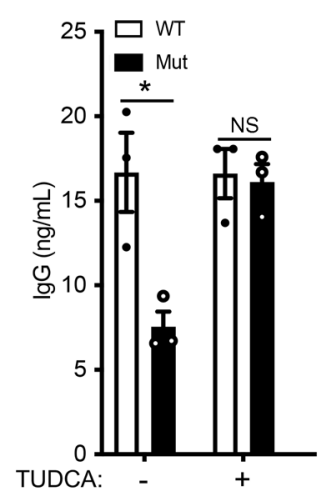

$\mathbf{L}$

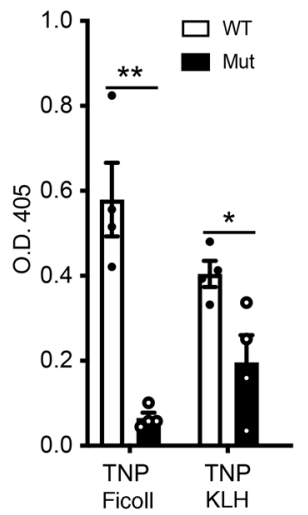

C

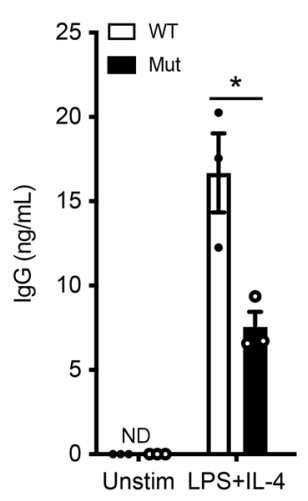

G
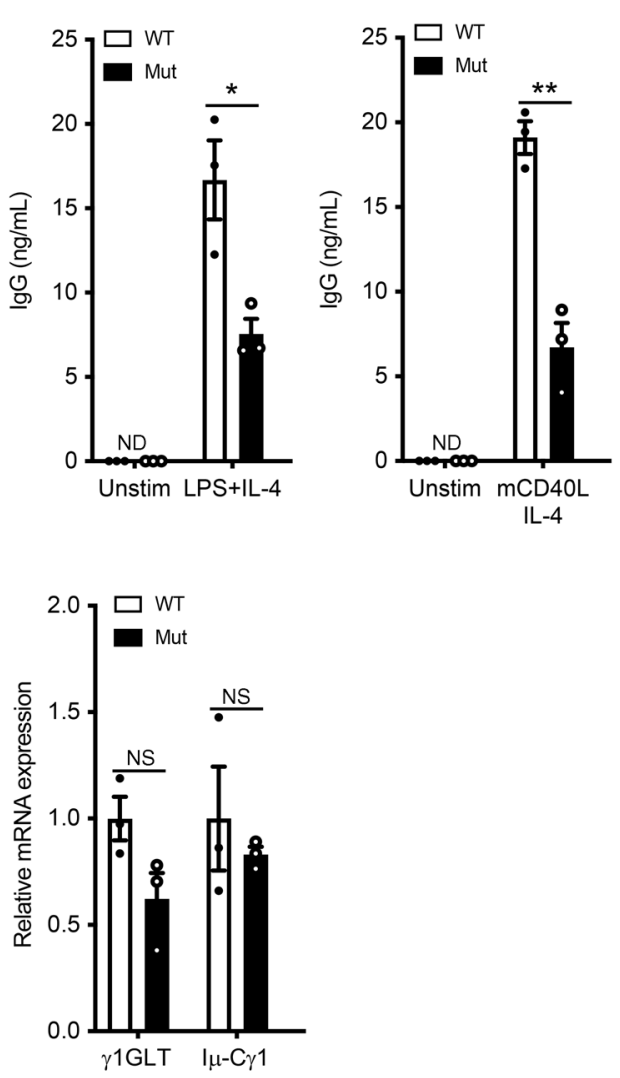

J
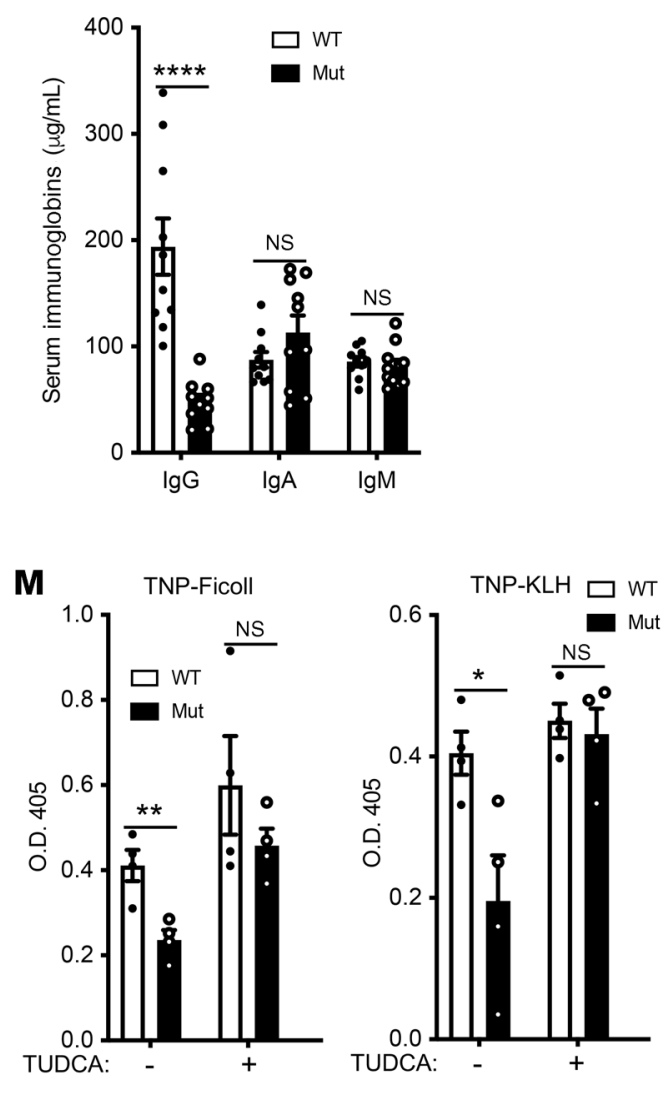
Figure 4. Defective B cell function in mutant mice. (A) Representative FACS of B cell proliferation following stimulation with LPS+IL-4 or $\mathrm{mCD} 40 \mathrm{~L}+\mathrm{IL}-4$ in mutant mice and WT controls. (B) Quantitation of the number of viable cells (left) and percentage of fixable viability dye negative (FVD-Annexin+) apoptotic cells (right) after LPS+IL-4 stimulation. (C) In vitro IgC secretion after LPS+IL-4 (left) or mCD4OL+IL-4 (right) stimulation of $B$ cells from mutant mice and WT controls. (D-C) Percentage of $\mathrm{B}_{22} \mathrm{O}^{\circ} \mathrm{CD} 138^{+}$plasmablasts (D), relative expression of Prdm1 mRNA (E), IgC1 secretion (F), and relative expression of $C \gamma 1$ germline and $I \mu-C \gamma 1$ mature transcripts (G) in LPS+IL-4 stimulated B cells from mutant mice and WT controls. (H and I) Effect of TUDCA on sXbp1 and Hspa5 mRNA expression (H) and in vitro IgG secretion (I) in LPS+IL-4 simulated B cell cultures from mutant mice and WT controls. (J) Serum immunoglobulin levels (IgG, IgA, and IgM) in 8- to 12-week-old mutant mice and WT controls; $n=$ 10 per group. (K) Percentage of B220-CD138 ${ }^{+}$plasma cells in the bone marrow of mutant mice and WT controls. (L) Anti-TNP antibody response 21 days after immunization with the TI antigen TNP-Ficoll or the TD antigen TNP-KLH in mutant mice and WT controls. $n=4$ mice per group. TNP-specific antibodies were not detected in unimmunized mice. (M) Anti-TNP antibody response 21 days after immunization with TNP-Ficoll or TNP-KLH in 8- to 12-week-old mutant mice and WT controls treated daily i.p. with TUDCA or PBS (-) as control. Experiments were performed independently twice with 4 mice per group for $\mathbf{L}$ and $\mathbf{M}$. Similar results were obtained in 3 independent experiments each with 3 mice per group for $\mathbf{A}-\mathbf{I}$ and $\mathbf{K}$. Columns and bars represent mean and SEM. ${ }^{*} P \leq 0.05$; ${ }^{*} P \leq 0.01$; ${ }^{* *} P \leq$ $0.001 ;{ }^{* * *} P \leq 0.0001,2$-tailed Student's $t$ test.

that resting mutant B cells exhibited sparse and short strands of ER compared with the abundant and longer ER strands in resting WT cells (Supplemental Figure 4, A-D). Similarly, LPS+IL-4 activated WT cells displayed ER expansion with longer and more elaborate membranous strands than mutant B cells (Supplemental Figure 4, E-H). Collectively, the results suggested that impaired retrieval of KDEL-bearing chaperones to the ER causes exaggerated ER stress in activated mutant B cells.

Strategies used to counterbalance ER stress include antioxidant response and autophagy. Transcriptomic analysis of LPS+IL-4 activated mutant and WT B cells and anti-CD3+antiCD28 stimulated $\mathrm{T}$ cells revealed comparable expression of 16 genes important for the antioxidant response (Supplemental Figure 4I). Expression of 22 genes important for autophagy was comparable in LPS+IL-4 stimulated mutant and WT B cells (Supplemental Figure 4J). Immunoblotting revealed comparable levels of the autophagosome marker LC3II between WT and mutant B cells cultured with LPS and chloroquine (Supplemental Figure 4K).

Defective B cell function in Copg1 ${ }^{\mathrm{K} 652 \mathrm{E}}$ mutant mice. The reduction in the KDEL-bearing chaperones in the ER of mutant B cells was expected to result in an accumulation of misfolded immunoglobulins in the ER and concomitantly diminished immunoglobulin secretion. Mutant B cells exhibited intact proliferation and cell viability in response to stimulation with LPS+IL-4 or mCD40L+IL-4 (Figure 4, A and B). However, they secreted significantly less IgG compared with WT B cells (Figure 4C). This was not simply due to a defect in IL-4 responsiveness, as mutant B cells also secreted significantly less IgG in response to LPS stimulation than WT controls (Supplemental Figure 5A). Plasmablast differentiation following LPS+IL-4 stimulation was comparable in B cells from mutants and WT controls, as determined by the percentages of $\mathrm{B} 22 \mathrm{O}^{\mathrm{lo}} \mathrm{CD} 138^{+}$plasmablasts and expression of $\operatorname{Prdm} 1$ encoding the transcription factor BLIMP1
(Figure 4, D and E). Expression of Prdm1 was minimal in resting WT and mutant B cells (Supplemental Figure 5B). Thus, the decreased IgG secretion by the mutant B cells was not due to defective plasmablast differentiation.

To determine whether the decreased IgG secretion by mutant B cells was due to defective IgG switching, we examined IgG1 isotype switching by purified naive B cells following LPS+IL-4 stimulation (36). IgG1 secretion was significantly reduced in mutant B cells compared with WT B cells (Figure 4F). IgG1 isotype switching by LPS+IL- 4 involves the induction of $\mathrm{C} \gamma 1$ germline transcription and expression of Aicda, which mediates class switch recombination, resulting in the expression of postswitch mature $\mathrm{I} \mu-\mathrm{C} \gamma 1$ transcripts (37). Expression of $\mathrm{C} \gamma 1$ germline and mature $\mathrm{I} \mu-\mathrm{C} \gamma 1$ transcripts was comparable in LPS+IL-4 stimulated mutant and WT B cells (Figure $4 G$ ).

The hydrophobic cell permeable bile acid tauroursodeoxycholic acid (TUDCA) binds to unfolded proteins in the ER, prevents their aggregation, and reduces ER stress (38). The addition of TUDCA to LPS+IL-4 stimulated cultures reduced sXbp1 and Hspa 5 mRNA expression in mutant B cells to levels comparable to those in WT B cells (Figure 4H). Importantly, it normalized IgG secretion by LPS+IL-4 stimulated mutant B cells, but had no significant effect on WT B cells (Figure 4I). These results suggest that the depletion of KDEL-bearing chaperones from the ER increases stress in this compartment and impairs IgG secretion in mutant B cells.

Serum IgG, but not IgA or IgM, was significantly decreased in mutant mice (Figure 4J), but the percentage of B220- $\mathrm{CD} 138^{\text {hi }}$ plasma cells in the live lymphocyte gates was comparable in bone marrow, and inguinal LNs of mutant mice and WT controls (Figure $4 \mathrm{~K}$ and Supplemental $5 \mathrm{C}$ ). Mutant mice had significantly reduced antigen-specific antibody responses to the T-independent (TI) antigen TNP-Ficoll and the T-dependent (TD) antigen TNP-KLH (Figure 4L). Analysis of popliteal LNs from mice immunized in the hock with TNP-KLH revealed that the mutant had normal percentages of $\mathrm{T}$ follicular helper ( $\mathrm{Tfh})$ cells $\left(\mathrm{CD} 4^{+} \mathrm{CXCR} 5^{+} \mathrm{PD} 1^{+}\right)$and germinal center (GC) B cells $\left(\mathrm{B} 22 \mathrm{O}^{+} \mathrm{GL7}^{+}\right.$) (Supplemental Figure 5D). Furthermore, immunofluorescence microscopy revealed that the numbers and sizes of GCs as determined by GL7 staining were comparable in the spleens of TNP-KLH immunized mutant mice and WT controls (Supplemental Figure 5E). Treatment throughout the immunization protocol with TUDCA $(500 \mathrm{mg} / \mathrm{kg}$ i.p. injection daily) normalized the IgG anti-TNP antibody response of mutant mice to TNP-Ficoll and TNP-KLH immunization, but had no significant effect on the antibody response of WT controls (Figure $4 \mathrm{M})$. Collectively, these results further suggest that the $\gamma 1-\mathrm{COP}$ mutation impairs IgG secretion in vitro and in vivo by depleting the ER of chaperones.

Pancreatic islet $\beta$ cells and hepatocytes are highly secretory cells. Serum levels of insulin, glucose, alanine aminotransferase (ALT), and alkaline phosphatase (ALP) were normal in mutant mice (Supplemental Figure 5, F and G). Expression of $\gamma 2$-COP in hepatocytes, as in B cells, showed no compensatory increase in the mutant, as it was comparable in mutant mice and WT controls (Supplemental Figure 5H). The selectivity of the secretory defect to plasma cells suggests that $\gamma 2$-COP may substitute for $\gamma 1$-COP in nonlymphoid cells. 
A

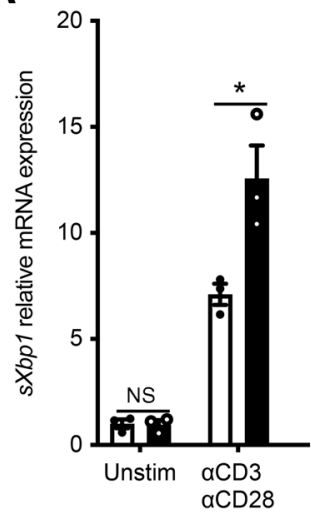

D

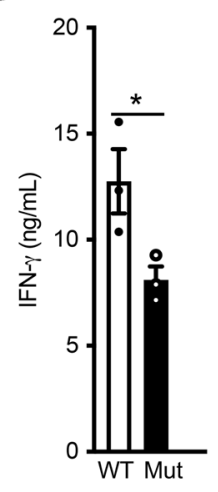

G

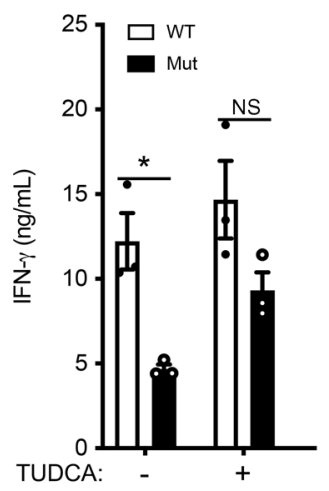

J

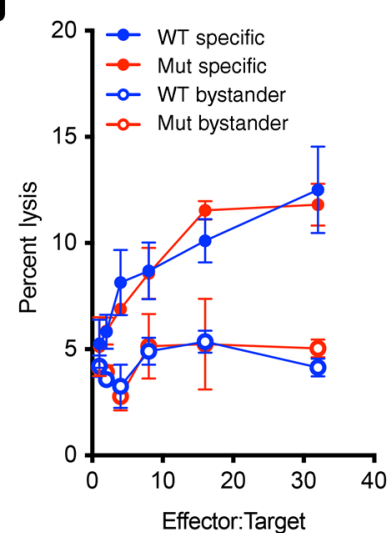

E
B
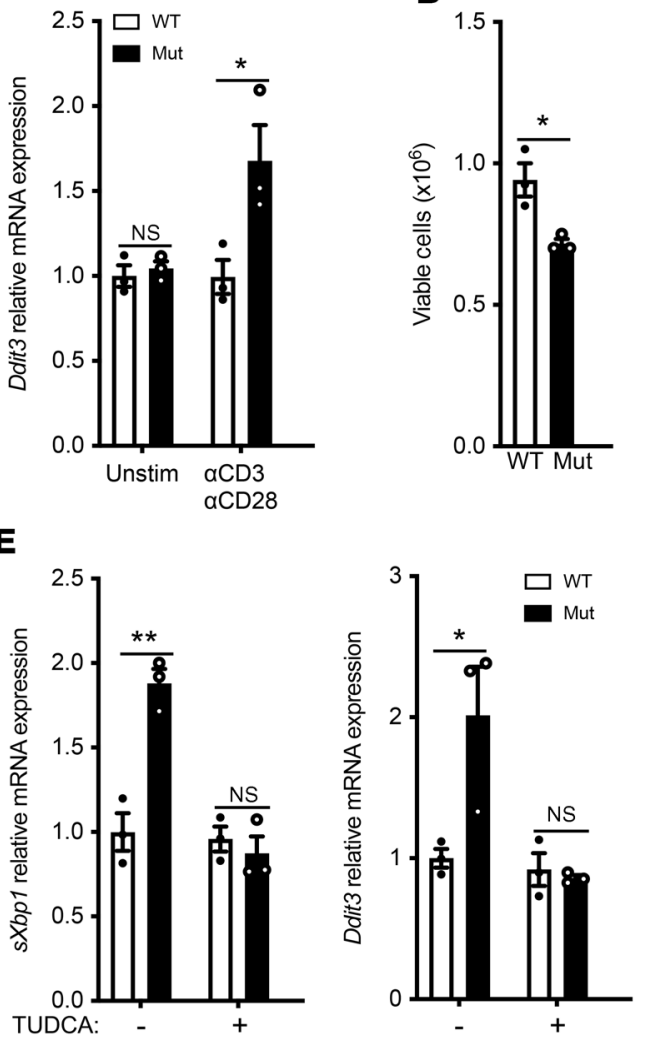

H
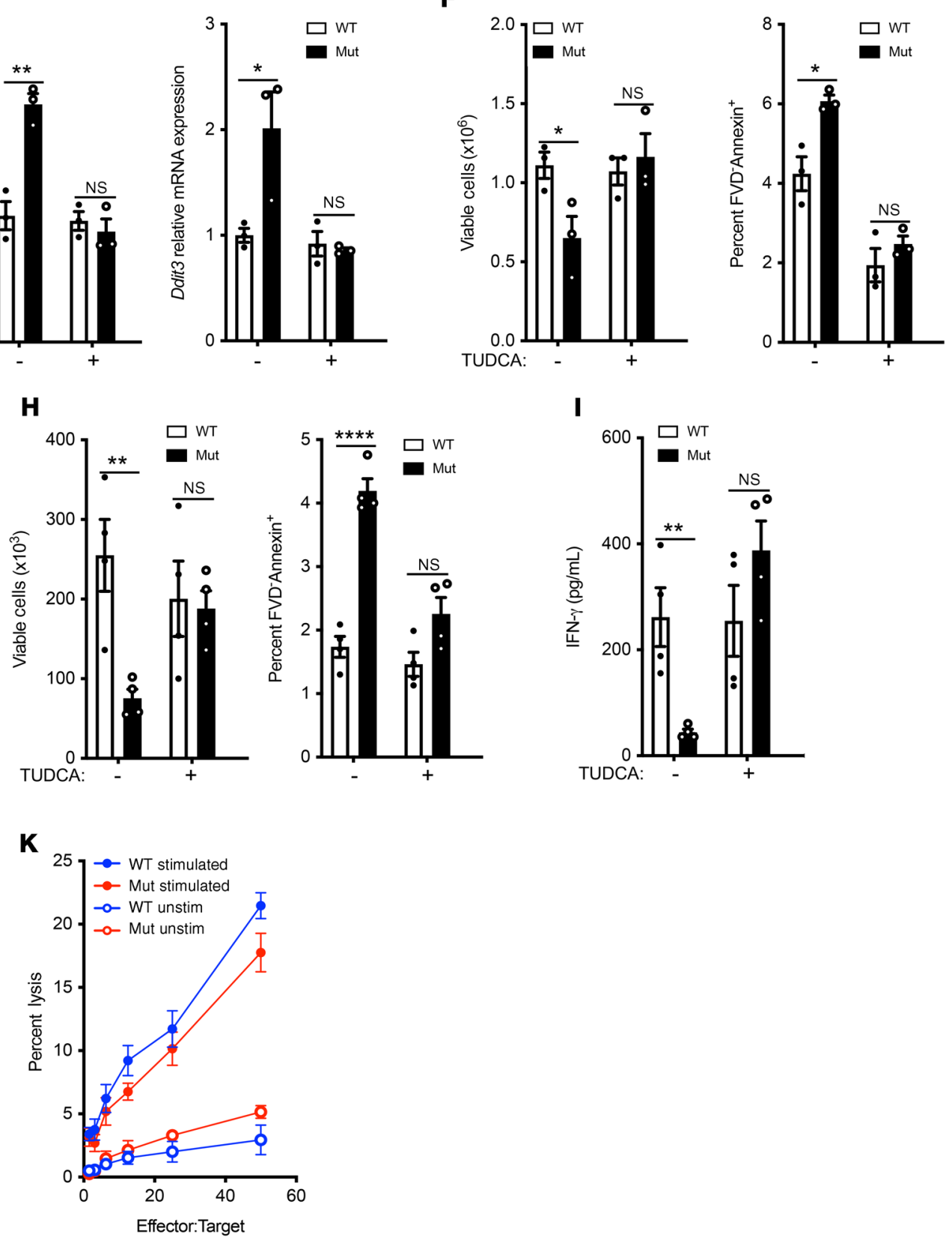
Figure 5. Mutant T cells have increased ER stress, decreased survival, and impaired function. (A-D) mRNA expression of ER stress response genes (A), numbers of viable T cells (B, left) and percentage of FVD-Annexin ${ }^{+}$ apoptotic cells among $\mathrm{CD}^{+} \mathrm{T}$ cells (B, right), representative histograms of CTV (C) and IFN- $\gamma$ secretion (D) after 3 days of T cell stimulation with $\alpha C D 3+\alpha C D 28$. (E-G) Effect of TUDCA addition on sXbp1 and Ddit3 mRNA expression (E) cell viability and apoptosis (F), and IFN- $\gamma$ secretion $(\mathbf{G})$ on $\alpha$ CD3 $+\alpha$ CD28 stimulated T cells. (H and I) Numbers of viable CD3 ${ }^{+} \mathrm{T}$ cells (H, left), percentage of FVD-Annexin ${ }^{+}$apoptotic cells among $\mathrm{CD}^{+}{ }^{+} \mathrm{T}$ cells (H, right), and IFN- $\gamma$ secretion (I) after 3 days of ex vivo stimulation of splenocytes from OVA-immunized mutant mice and controls with OVA in the presence or absence of TUDCA. $n=4$ independent experiments, 5 mice per group. (J) T cell allocytotoxicity in mutant and WT C57BL/6 (H2b) mice. Splenocytes were stimulated with mitomycin C-treated BALB/C $\left(\mathrm{H} 2^{\mathrm{d}}\right)$ splenocytes for 5 days, and cytotoxicity was measured in a 4-hour assay with P815 $\left(\mathrm{H} 2^{\mathrm{d}}\right)$ and EL4 $\left(\mathrm{H} 2^{\mathrm{b}}\right)$ cells. $n=2$ independent experiments each with 3 mice per group. (K) NK cell cytotoxicity in mutant and WT splenocytes. Mice were treated with either Poly(I:C) i.p. or PBS 18 hours prior to harvest. Cytotoxicity of WT and mutant splenic NK cells was measured in a 4-hour assay against ${ }^{51} \mathrm{Cr}$-labeled YAC-1 cells. $n=2$ experiments each with 3 mice per group. Similar results were obtained in 3 independent experiments each with 3 mice per group for A-G. Columns and bars represent mean and SEM. ${ }^{*} P \leq 0.05 ;{ }^{* *} P \leq 0.01 ;{ }^{* * *} P \leq 0.001$, 2 -tailed Student's $t$ test $(\mathbf{A}-\mathbf{D})$; Holm-Šídák test to control for multiple comparisons (E-I); 2-way ANOVA (J); Mann-Whitney U test (K).

$T$ cells from Copg1 ${ }^{\mathrm{K} 652 \mathrm{E}}$ mutant mice have increased ER stress, decreased survival, and impaired IFN- $\gamma$ secretion. Freshly isolated splenic T cells from mutant mice and WT controls cells had comparable levels of $s X b p 1$ and Ddit3 mRNA. Stimulation with antiCD3+anti-CD28 increased expression of both genes, but their levels were significantly higher in mutant $\mathrm{T}$ cells compared with controls (Figure 5A). Increased CHOP expression drives apoptosis and cell death in $\mathrm{T}$ cells (22). Following anti-CD3+anti-CD28 stimulation, mutant $\mathrm{CD}^{+} \mathrm{T}$ cells had reduced viability and increased apoptosis compared with WT controls (Figure 5B). Both CD4 ${ }^{+}$ and $\mathrm{CD}^{+}$subpopulations were affected (Supplemental Figure $6 \mathrm{~A})$. $\mathrm{T}$ cell proliferation and upregulation of surface expression of the activation markers CD25, CD69, and CD40L following antiCD3+anti-CD28 stimulation were comparable between mutant and WT controls (Figure 5C and Supplemental 6B). IFN- $\gamma$ secretion was significantly reduced in cultures of anti-CD3+anti-CD28 stimulated mutant T cells compared with WT controls (Figure 5D). The reduction in IFN- $\gamma$ secretion by the mutant was commensurate with the reduction in $\mathrm{T}$ cell numbers. IFN- $\gamma$ secretion on a per cell basis as well as intracellular IFN- $\gamma$ expression were comparable in mutant and WT T cells (Supplemental Figure 6, C and D). These results suggest that the decreased IFN- $\gamma$ secretion by the mutant's activated T cells was secondary to their reduced viability.

We investigated whether the impaired survival and function of activated mutant $\mathrm{T}$ cells was due to increased ER stress. The addition of TUDCA to anti-CD3+anti-CD28 stimulated $\mathrm{T}$ cell cultures reduced $s X b p 1$ and Ddit 3 mRNA expression in mutant $\mathrm{T}$ cells to levels comparable to those in WT controls (Figure 5E). Importantly, the addition of TUDCA to anti-CD3+anti-CD28 stimulated cultures restored the survival and viability of mutant $\mathrm{CD}^{+}, \mathrm{CD}^{+}$, and $\mathrm{CD} 8^{+} \mathrm{T}$ cells, but had no significant effect on WT T cells (Figure 5, F and G, and Supplemental Figure 6, E and F)

We also examined the impact of mutant $\gamma 1-\mathrm{COP}$ on the antigen-specific $\mathrm{T}$ cell response to i.p. immunization with OVA. Fol- lowing stimulation of splenocytes with $\mathrm{OVA}, \mathrm{CD}^{+} \mathrm{T}$ cells from the mutants had significantly reduced viability, increased apoptosis, and decreased IFN- $\gamma$ secretion compared with WT controls (Figure 5, $\mathrm{H}$ and I). Treatment of mutant mice with TUDCA throughout the OVA immunization protocol reversed these defects (Figure 5, H and I). Collectively, these results indicate that increased ER stress impairs the survival and function of activated mutant $\mathrm{T}$ cells.

Cytotoxic $\mathrm{CD}^{+} \mathrm{T}$ cells and NK cells are important for eliminating virally infected cells (39). Given the patients' persistent CMV and EBV viremias, we examined cytotoxic $T$ cell and NK cell activities of $\gamma 1$-COP mutant mice. $T$ cell cytotoxicity against allogeneic targets as well as NK cell cytotoxicity were comparable in mutants and WT controls (Figure 5, J and K). These findings indicate that the $\operatorname{Copg}^{\mathrm{K}}{ }^{622 E}$ mutation does not impair intrinsic $\mathrm{T}$ cell or NK cell cytotoxic activity.

Copg1 ${ }^{K 652 E}$ mutant mice recapitulate the patients' phenotype under conditions of natural microbial exposure. Unlike the patients, mutant mice grew normally, demonstrated no $\mathrm{T}$ cell lymphopenia, had normal $\mathrm{T}$ cell proliferation to anti-CD3+anti-CD28, and had hypogammaglobulinemia. To determine whether these differences might be due to restricted exposure of mice to pathogens under specific pathogen-free (SPF) conditions, we first examined the effect of chronic infection with lymphocytic CMV (LCMV). Infection with LCMV clone 13 resulted in no significant differences between mutant and WT mice as to body weight or spleen T cell counts, although serum IgG rose to the levels in WT controls (Supplemental Figure 7, A-C). Multiple studies have highlighted that exposure to a diversity of natural microbes, rather than a single pathogen, recapitulates the immunologic challenges encountered by humans (40-43). To investigate the effect of exposure to a broad range of natural microbes, mutant mice and WT controls ( $n=8$ each) were cohoused with pet store mice for 22 days in 2 independent experiments. Mouse PCR Rodent Infectious Agent (PRIA) panels performed on stools on day 22 revealed that in each experiment, mutant mice and WT controls had acquired a profile of microbes similar to that of the pet store mice (Supplemental Table 3). Cohoused WT mice dropped their body weight to a nadir of $90 \%$ ( $10 \%$ loss) on day 5 , but quickly regained their original body weight by day 7 and maintained it throughout the experiment (Figure 6A). Cohoused mutant mice steadily lost body weight and reached a mean of $70 \%$ of their prehousing body weight on day 22, when the experiment was terminated (Figure 6A). Five of eight became hunched and developed ruffled hair.

Serum levels of TNF- $\alpha$ and IFN- $\gamma$ were modestly increased on day 11 and returned to normal on day 22 in cohoused WT mice, but were significantly more elevated on both days in the cohoused mutant mice, while IL- 6 was markedly elevated in the mutants on day 22 (Figure 6B). Following exposure for 22 days to pet store mice, mutant mice demonstrated significantly increased spleen expression of $X b p 1$ and $s X b p 1$ compared with WT controls (Figure 6C). Importantly the mutants demonstrated decreased numbers of splenic $\mathrm{CD}^{+}, \mathrm{CD}^{+}$, and $\mathrm{CD} 8^{+}$cells compared with WT controls (Figure 6D). Following cohousing, the percentage and surface expression of $\mathrm{CD} 9^{+}$and of $\mathrm{CD} 44^{+}$, but not of $\mathrm{PD} 1^{+}$, cells were significantly higher in the mutants compared with WT controls (Supplemental Figure 8, A and B). Mutant mice cohoused with pet store mice for 22 days demonstrated substantially reduced anti- 

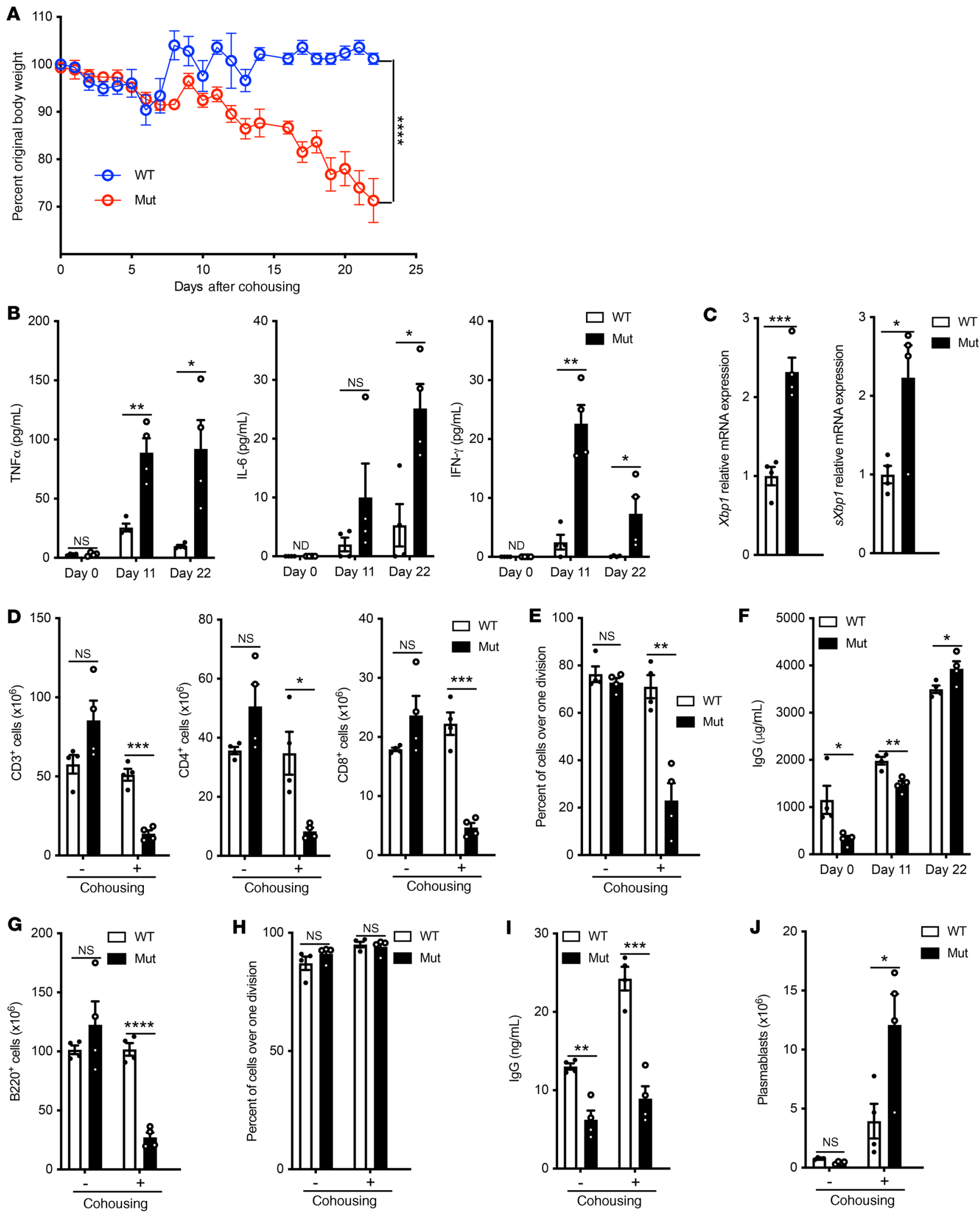
Figure 6. Effect of cohousing Copg $1^{K 652 E}$ mice with pet store mice. (A) Weight of mice after cohousing with pet store mice for 22 days, as percentage of weight at day 0. (B) TNF- $\alpha$, IL- 6 and IFN- $\gamma$ serum levels. (C) Xbp1 and $s$ Xbp1 expression by splenocytes from mice after cohousing with pet store mice for 22 days. Values relative to gene expression of cohoused WT controls. (D) Numbers of viable $\mathrm{CD}^{+}, \mathrm{CD}^{+}$, and $\mathrm{CD} 8^{+} \mathrm{T}$ cells in the spleens of mice housed in SPF conditions or cohoused for 22 days with pet store mice. (E) Proliferation of CTV loaded purified splenic T cells in response to $\alpha \mathrm{CD} 3+\alpha \mathrm{CD} 28$ stimulation in WT and mutant mice housed in SPF conditions or cohoused for 22 days with pet store mice. Results are expressed as the percentage of cells that have undergone more than 1 cell division as determined by dilution of the CTV dye. (F) Serum IgG levels on days 0,11 , and 22. (G) Numbers of viable B220+ cells in the spleens of WT and mutant mice housed in SPF conditions or cohoused for 22 days with pet store mice. ( $\mathbf{H}$ and $\mathbf{I})$ Proliferation $(\mathbf{H})$ and in vitro IgG secretion (I) of purified splenic B cells in response to LPS+IL-4 in WT and mutant mice housed in SPF conditions or cohoused for 22 days with pet store mice. (J) Numbers of $B 220^{10} \mathrm{CD} 138^{+}$plasmablasts in the spleens of WT and mutant mice housed in SPF conditions or cohoused for 22 days with pet store mice. Results in A and J were pooled from 2 independent experiments each with 4 mice/ group. Columns and bars represent mean and SEM. ${ }^{*} P \leq 0.05 ;{ }^{*} P \leq 0.01$; ${ }^{* * *} P \leq 0.001 ;{ }^{* * *} P \leq 0.0001,2$-way ANOVA (A), 2-tailed Student's $t$ test (B), and Holm-Šídák test to control for multiple comparisons (C and J).

CD3+anti-CD28 driven T cell proliferation compared with WT controls (Figure 6E). This is in contrast to the normal T cell proliferation in mutant mice kept under SPF conditions (Figure 5C).

Serum IgG levels, which were decreased in mutant mice raised under SPF conditions, significantly increased in mutant and WT mice cohoused with pet store mice and became comparable in the 2 groups on day 22 of cohousing (Figure 6F). Cohousing of mutant mice and WT controls with pet store mice for 22 days caused a significant reduction in the numbers of splenic $\mathrm{B}^{2} 2 \mathrm{O}^{+}$cells (Figure $6 \mathrm{G}$ ), while having no effect on B cell proliferation and in vitro IgG secretion in response to LPS+IL-4 stimulation (Figure 6, $\mathrm{H}$ and I). Importantly, cohousing caused a marked increase in the numbers of splenic $\mathrm{B} 22 \mathrm{O}^{10} \mathrm{CD} 138^{+}$plasmablasts in the mutants compared with WT controls (Figure 6J). Increased serum IL-6 levels may have contributed to the increase in plasmablasts in the mutants and thereby to the rise in their serum IgG to levels comparable to those of WT controls. Collectively, the results demonstrate that, upon exposure to the microbiota of pet store mice, mutant mice develop cachexia, secondary lymphopenia, impaired $\mathrm{T}$ cell proliferation, and hypergammaglobulinemia, recapitulating the findings in the patients.

Treatment of Copg1 ${ }^{K 652 E}$ mutant mice with TUDCA partially reverses the phenotype caused by microbial exposure. We investigated whether increased ER stress contributes to the phenotype triggered in Copg1 ${ }^{K 652 E}$ mutant mice by cohousing with pet store mice. TUDCA or vehicle control was administered daily to the mutants for 21 days starting on day 0 of cohousing. In the same experiment, a group of WT mice were cohoused with pet store mice as control. Treatment of cohoused mutant mice with TUDCA resulted in significantly less reduction in body weight compared with treatment with vehicle control (Figure 7A). It also attenuated the increase in serum levels of TNF- $\alpha$ and IFN- $\gamma$, but had no effect on serum IL- 6 levels (Figure 7B). TUDCA treatment of cohoused mutant mice significantly decreased $s X b p 1$ expression by splenocytes (Figure 7C). Importantly, it reversed their splenic $\mathrm{CD}^{+}, \mathrm{CD}^{+}$, and $\mathrm{CD}^{+}$ cell lymphopenia (Figure 7D). Moreover, it corrected their defec- tive $\mathrm{T}$ cell proliferation to anti-CD3+anti-CD28 (Figure 7E). TUDCA treatment of cohoused mutant mice had no significant effect on their serum IgG levels (Figure 7F) or on plasmablast expansion in their spleen (Figure 7G), consistent with the absence of a detectable effect on serum IL- 6 levels. These data suggest that increased ER stress contributes to the adverse effects of exposure of the mutant to multiple pathogens.

\section{Discussion}

We have identified a homozygous missense mutation in COPG1 as a cause of CID. The Copg1 ${ }^{K 62 E}$ mutation disrupts the binding of the KDELR by coatomer. This results in impaired retrograde COPI-mediated transport of the KDELR from the Golgi to the ER, reducing the level of KDEL-bearing chaperones in the ER, which leads to increased ER stress in activated T and B cells. We modeled the disease in mice. These recapitulated the patients' findings upon exposure to natural microbes. Importantly, relieving ER stress with TUDCA corrected the immune defects of the mutants and reversed to a large extent the phenotype they acquired following exposure to pet store mice, demonstrating that increased ER stress underlies the immunologic defect in $\gamma 1$-COP deficiency.

The failure of coatomer isolated from the liver of Copg1 ${ }^{\mathrm{K} 652 \mathrm{E}}$ mice to interact directly with the KDELR demonstrates that $\gamma 1$-COP is the subunit critical for COPI interacting with the KDELR and thus for COPI-mediated retrograde trafficking of KDEL motifcontaining ER chaperones. The $\gamma 1$-COP paralog, $\gamma 2$-COP, did not compensate for the lost function of mutant $\gamma 1-\mathrm{COP}$ in the retrograde trafficking of KDELR. The 2 paralogs have poor sequence homology in the region surrounding the $\mathrm{K} 652$ residue mutated in $\gamma 1$-COP. Furthermore $\gamma 1$-COP-containing COPI complexes are localized to the early/cis-Golgi, while $\gamma 2$-COP-containing COPI complexes are found primarily in the late Golgi (44), suggesting that the 2 paralogs have distinct functions. $\gamma 2$-COP may compensate for the impaired $\gamma 1-\mathrm{COP}$ function in nonlymphoid tissues.

Defective retrograde trafficking of the KDELR resulted in depletion of KDEL motif-containing chaperones from the ER of activated B cells from $\operatorname{Copg}^{\mathrm{K}}{ }^{652 E}$ mutant mice, resulting in increased ER stress and activation of the UPR. Increased ER stress was detected only after activation of mutant $B$ and $T$ cells, suggesting that the impact of the defect in retrograde trafficking on ER stress becomes evident only during robust protein secretion.

Under SPF conditions, the mutant mice demonstrated hypogammaglobulinemia, impaired IgG secretion in vitro, and defective antibody responses to TI and TD antigens. Class-switch recombination and expression of switched transcripts, plasmablast differentiation in vitro, and plasma cell numbers in the bone marrow were all normal in the mutant, indicating a defect in IgG secretion. The chemical chaperone TUDCA restored IgG secretion and antibody responses in the mutant, suggesting that these defects were due to IgG misfolding in the ER. Data in the literature indicate that, although the serum level of IgG is 3 times higher than that of IgM and IgA, the percentage of bone marrow plasma cells that express intracellular IgG is 4- to 6-fold lower that of plasma cells that express IgM or IgA (45). This suggests that the rate of immunoglobulin secretion is higher in IgG-secreting plasma cells. This may explain the higher susceptibility of IgG secretion to the depletion of ER chaperones in the mutant. 
A
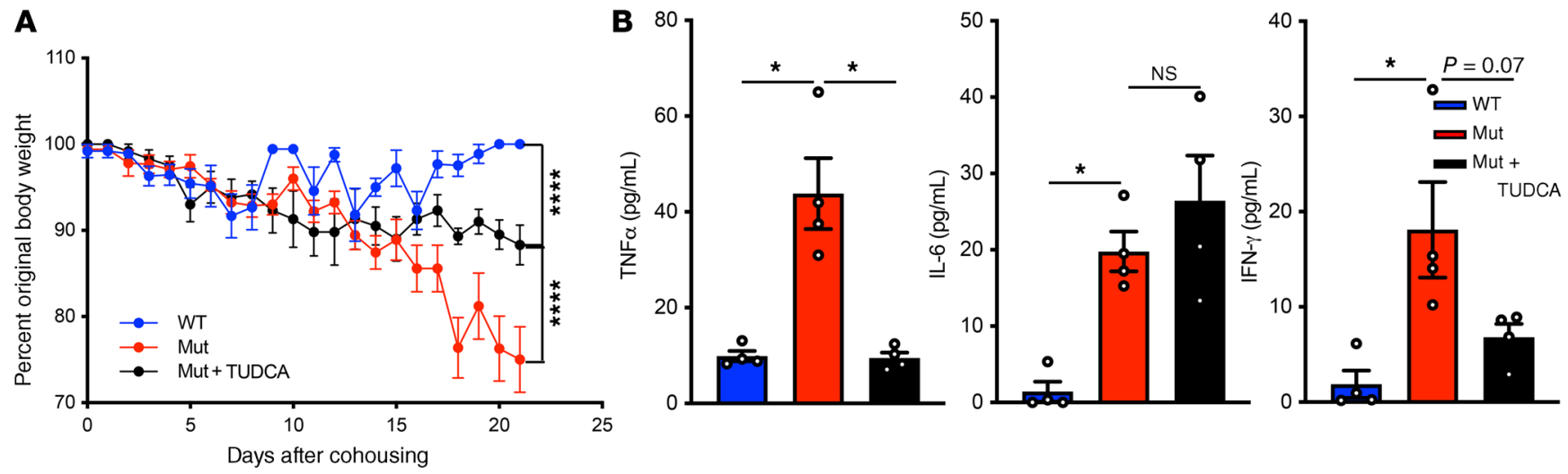

C

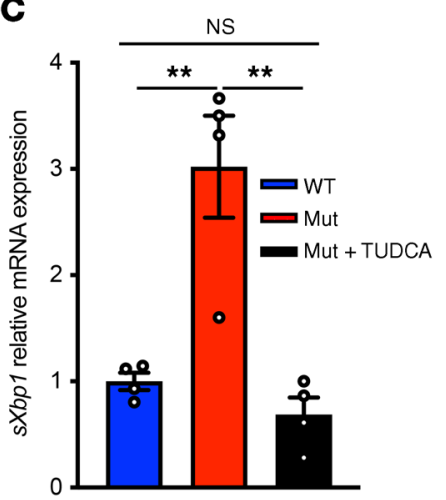

D
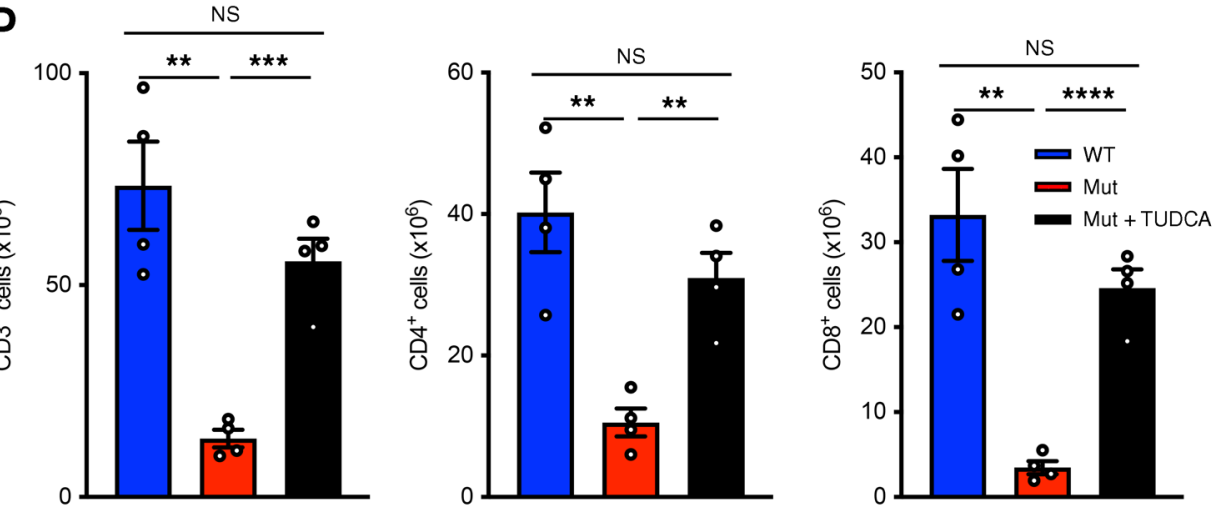

E

$\mathbf{F}$

G


Figure 7. Treatment of Copg ${ }^{1652 E}$ mutant mice with TUDCA partially reverses the phenotype induced by microbial exposure. Mutant mice received TUDCA or vehicle daily for 21 days starting on day 0 of cohousing. In the same experiment, a group of WT mice were cohoused with pet store mice as control. (A) Weight expressed as percentage of body weight at day 0 of cohousing. (B) Serum TNF- $\alpha$, IL-6, and IFN- $\gamma$ levels on day 21. (C) Splenocyte expression of $s$ Xbp1 on day 21. Values are relative to gene expression of cohoused WT mice. (D) Numbers of viable CD3+, $C D 4^{+}$, and CD8 ${ }^{+}$T cells in the spleens on day 21. (E) Proliferation of purified splenic T cells in response to $\alpha \mathrm{CD} 3+\alpha \mathrm{CD} 28$ stimulation on day 21. (F and $\mathbf{G})$ Serum IgG levels $(\mathbf{F})$ and numbers of B220 ${ }^{\circ} \mathrm{CD} 138^{+}$plasmablasts in the spleens (G) on day 21. Results for $\mathbf{A}$ and $\mathbf{G}$ were pooled from 2 independent experiments each with 4 mice/group. Columns and bars represent mean and SEM. ${ }^{*} P \leq 0.05 ;{ }^{* *} P \leq 0.01 ;{ }^{* *} P \leq 0.001 ;{ }^{* * *} P \leq 0.0001$, 2-way ANOVA (A); Holm-Šídák test to control for multiple comparisons (B and $\left.\mathbf{G}\right)$.

T cells from Copg1 ${ }^{1652 E}$ mice raised under SPF conditions had decreased viability, increased apoptosis, and decreased IFN- $\gamma$ secretion following polyclonal and antigen-specific restimulation in vitro. These abnormalities were reversed by TUDCA, indicating that they were driven by increased ER stress. Activation of the PERK pathway and expression of CHOP in ER-stressed T cells, but not B cells, likely explains the selective susceptibility of $\gamma 1-\mathrm{COP}-$ deficient $\mathrm{T}$ cells to apoptosis and cell death upon activation. The proliferation by live mutant $\mathrm{T}$ cells following anti-CD3+anti-CD28 stimulation and the allocytoxicity of these cells against allogeneic targets were not affected, indicating that the $\gamma 1$-COP mutation did not globally impair $\mathrm{T}$ cell function.

$\mathrm{P} 1-\mathrm{P} 4$ presented with infections and demonstrated CD4 lymphopenia as well as impaired T cell proliferation. Notably, P5 had normal $\mathrm{T}$ cell numbers at birth, However, by 9 months of age, he developed severe $\mathrm{CD} 4^{+} \mathrm{T}$ cell lymphopenia and impaired $\mathrm{T}$ cell proliferation in the context of respiratory infections and CMV viremia. In contrast, under SPF conditions, Copg1 ${ }^{K 62 E}$ mice exhibited 
normal growth, $T$ cell numbers, and proliferation and reduced $\operatorname{Ig} G$ levels. However, following microbial exposure through cohousing with pet store mice, mutant mice demonstrated increased ER stress in their splenocytes and developed a phenotype that resembled that of the patients, including cachexia, T cell lymphopenia, severely impaired $\mathrm{T}$ cell proliferation, and hypergammaglobulinemia. The increased serum levels of TNF- $\alpha$, IFN- $\gamma$, and IL- 6 in the mutants may have contributed to their cachexia, $\mathrm{T}$ cell lymphopenia, and plasmacytosis, respectively. Cytokines and ER stress reinforce each other. Increased ER stress activates transcription factors, including NF- $\mathrm{KB}$ and $\mathrm{AP}-1$, that drive the production of inflammatory cytokines (46). In turn, high levels of inflammatory cytokines can increase ER stress (47). Sustained increase in ER stress can result in cell death to which activated $\mathrm{T}$ cells are more susceptible than activated B cells because of their higher expression of CHOP. Administration of TUDCA to mutant mice reversed the exaggerated ER stress and, partially, the phenotype induced by exposure to pet store mice. It also corrected the $\mathrm{T}$ cell lymphopenia and proliferative defect induced by this exposure. These findings underscore the potential contribution of ER stress triggered by microbial exposure to disease manifestations in the patients.

Mutations in other subunits of coatomer have been previously reported. Variants in $\alpha$-COP have been associated with COPA syndrome characterized by arthritis, high-titer autoantibodies to blood cells, and interstitial lung disease (48). None of these features were present in the $\gamma 1$-COP-deficient patients. Mutations in $\delta$-COP present with craniofacial syndrome, and a hypomorphic mutation in $\beta^{\prime}-\mathrm{COP}$ is associated with human microcephaly (49, $50)$. Both of these abnormalities were absent in our patients. In the previous studies, the effects of human mutations in $\alpha$-COP, $\beta^{\prime}$-COP, or $\delta$-COP on COPI-mediated trafficking were not scrutinized, and none have been associated with immune deficiency.

To our knowledge, $\gamma 1-\mathrm{COP}$ deficiency is the first known primary immunodeficiency disease in which the underlying cause is the defective retrograde trafficking of the KDELR, leading to increased ER stress. The deficiency impairs immune function by causing a depletion of ER chaperones from the ER, which interferes with IgG secretion by plasma cells and causes excessive ER stress that results in the death of activated $\mathrm{T}$ cells. This renders the patients susceptible to persistent infections, which results in $\mathrm{T}$ cell depletion. The persistent microbial load and excessive production of inflammatory cytokines can cause cachexia and ultimately death.

In summary, our findings demonstrate the importance of the $\gamma 1$-COP subunit in COPI binding to the KDELR, establish a critical role for ER homeostasis in adaptive immunity, and illustrate the value of studying mouse models of immunodeficiency under conditions of natural microbial exposure. They also suggest that the chemical chaperone that reduces ER stress, TUDCA, may be of value in treating patients with $\gamma 1-\mathrm{COP}$ deficiency and other immune deficiencies in which increased ER stress may play a role.

\section{Methods}

Human studies. Studies on patient and controls were performed as previously described (51). In brief, human PBMCs were isolated from heparinized blood using Ficoll-Hypaque (GE Healthcare). Cells were suspended in RPMI-1640 containing 10\% heat-inactivated FCS (HyClone), $2 \mathrm{mM}$ L-glutamine, $50 \mu \mathrm{g} / \mathrm{mL}$ streptomycin, and $100 \mathrm{U} /$
$\mathrm{mL}$ penicillin (medium). Fibroblast cell lines were established from skin biopsies following standard procedures and were used after at least 4 or 5 passages. PBMCs $\left(1 \times 10^{6}\right.$ and $1.5 \times 10^{6}$ cells $/ \mathrm{mL}$ for proliferation and immunoglobulin production, respectively) were cultured with medium, PHA (1 $\mu \mathrm{g} / \mathrm{mL}$; MilliporeSigma), or $\alpha$-CD3 $(100 \mathrm{ng} / \mathrm{mL}$; OKT3, eBioscience) for 4 days or with $\alpha$-CD $40(5 \mu \mathrm{g} / \mathrm{mL}$; 626.1) plus recombinant IL-4 (5 ng/mL; R\&D Systems) for 14 days. Proliferation was assayed by measuring the incorporation of $\left[{ }^{3} \mathrm{H}\right]$-thymidine added for the last 16 hours of culture. IgG was measured in culture supernatants by ELISA.

WES. Ten micrograms of genomic DNA were isolated from peripheral blood samples using the Gentra Puregene Blood Kit (QIAGEN) per the manufacturer's protocol. WES of 2 patients and the mother was performed with an Illumina HiSeq-2000. The paired-end Illumina libraries were captured in solution according to the Agilent Technologies SureSelect protocol with $101 \mathrm{bp}$ read length. Sequence data were mapped to the human reference genome (hg-19, NCBI37) using the Burrows-Wheeler Alignment method at default settings. Variants were identified with the Genome Analysis Toolkit, SAMtools, and Picard Tools (http://broadinstitute.github.io/picard/). Variants with a read coverage of less than 2 times or Phred-scaled, single-nucleotide polymorphism quality of less than 20 were discarded.

Autozygosity analysis and Sanger sequencing. DNA samples were genotyped on an Axiom Chip platform per the manufacturer's protocol (Affymetrix). Runs of homozygosity (ROH) greater than $2 \mathrm{Mb}$ that spanned 107 SNPs were used as surrogates of autozygosity, i.e., identical by state, given the consanguineous nature of the family using autoSNPa (52). The entire set of autozygosity blocks (autozygome) was determined for each affected member, and potential overlaps between the autozygomes of affected members were pursued. Sanger sequencing was performed as detailed in the Supplemental Methods.

Generation of COPG1 ${ }^{K 652 E}$ mice. sgRNA design was facilitated though the CRISPR Design tool (crispr.mit.edu) to minimize off-target effects. A guide was selected that was predicted to generate a double-stranded break 4 nucleotides from the intended c.1954A>G p.K652E mutation. A 160 bp repair template was designed that included the c.1954A $>$ G mutation as well as synonymous c.1944C $>$ T change in the protospacer adjacent motif to prevent repair template targeting by the CAS9 nuclease. Zygotes were microinjected with CAS9 mRNA (System Biosciences), a single-stranded repair template oligo (PAGE Ultramer from Integrated DNA Technologies), and the sgRNA. For sgRNA synthesis, the T7 promoter sequence was added to sgRNA template/forward primer and the IVT template generated by PCR amplification. The T7-sgRNA PCR product was purified and used as the template for IVT using MEGAshortscript T7 Kit (Life Technologies). The sgRNA was purified using the MEGAclear Kit (Life Technologies). Aliquots from an IVT reaction were separated on agarose gel to assess reaction quality.

Immunoblotting. Immunoblotting was performed on cell lysates using standard methods; antibodies are described in the Supplemental Methods.

Flow cytometry. Standard flow cytometric methods for the staining of cell-surface and intracellular proteins and the antibodies used are described in the Supplemental Methods.

COPI retrograde transport assay. The VSVG-ts045-KDELR construct in mammalian expression vector has been described $(27,30)$. The retrograde COPI transport assay was performed as previously 
described $(28,53)$. In brief, cells were transfected with VSVG-ts045KDELR for 48 hours. Cells were then incubated at $40^{\circ} \mathrm{C}$ for 18 hours to accumulate the COPI cargo at the ER. Cells were then incubated at $32^{\circ} \mathrm{C}$ for 2 hours for the synchronized accumulation of the COPI cargo at the Golgi. Subsequently, the temperature was shifted to $40^{\circ} \mathrm{C}$ to allow 1 round of retrograde transport of the COPI cargo from the Golgi to the ER. To quantify this transport, the colocalization of the COPI cargo with a Golgi marker (GM130) was assessed over time using confocal microscopy, as described in further detail below.

Confocal microscopy. Colocalization studies in fibroblasts and activated B cells and quantification of the results were performed as described in the Supplemental Methods.

GST pulldown assay. Pulldown assays to examine the direct binding of coatomer to different partners have been described $(31,33)$. In brief, plasmids encoding for GST fusions of KDELR, Wbp1, and ARFGAP1 in bacterial expression vectors were expressed in bacteria (BL21 cell) with ITPG induction. After lysis in buffer (20 mM Tris [pH 8.0], $100 \mathrm{mM} \mathrm{NaCl}, 1 \mathrm{mM}$ EDTA, $1 \%$ Triton X-100, $1 \mathrm{mg} / \mathrm{ml}$ of lysozyme and protease inhibitor), the cell lysate was incubated with Glutathione Sepharose beads (GE Healthcare). GST fusions on beads were then incubated with purified coatomer $(2.5 \mathrm{nM})$ at $4^{\circ} \mathrm{C}$ for 1 hour using an incubation buffer (25 mM Hepes [pH 7.2], $50 \mathrm{mM} \mathrm{KCl,} 2.5 \mathrm{mM}$ $\mathrm{Mg}[\mathrm{OAc}] 2$, and $0.5 \% \mathrm{NP}-40)$. Beads were then rinsed twice with the incubation buffer and then analyzed by SDS-PAGE followed by immunoblotting, Coomassie staining, or silver staining.

FLIM. Interactions were monitored using time-correlated single-photon counting fluorescence lifetime image microscopy analysis (TCSPC-FLIM). In brief, coatomer was detected with a mouse anti-coatomer antibody, which was coupled to a secondary antibody conjugated to Alexa Fluor 488 (donor fluorophore). The interacting partner, VSVG-ts045-KDELR or ARFGAP1, was detected using a rabbit antiMyc antibody (directed against the Myc tag appended to VSVG-ts045KDELR) or a rabbit anti-ARFGAP1 antibody, respectively. These rabbit antibodies were coupled to a secondary antibody conjugated to Alexa Fluor 594 (acceptor fluorophore). The baseline lifetimes of the donor fluorophore were calculated by single-exponential decay fitting of fluorescence emission in the absence of the acceptor fluorophore. For samples that contained both donor and acceptor fluorophores, lifetimes were fitted to a biexponential decay with lifetime of 1 component fixed to the lifetime of the donor-only condition. The interactions of coatomer with VSVG-ts045-KDELR or ARFGAP1 were quantified at the Golgi region of cells.

Cell stimulation and determination of secreted immunoglobulins and cytokines. Studies were performed in isolated splenic B and T cells as detailed in the Supplemental Methods.

Gene expression analysis. Standard methods for mRNA isolation, cDNA preparation, quantitative PCR (qPCR) analysis, and the primers used are detailed in the Supplemental Methods.

Immunizations and TUDCA treatment. Specific antibody responses to immunization were studied by immunizing 8- to 12-week-old Cop- $g 1^{K 652 E}$ and WT (C57BL/6) mice i.p. with $10 \mu \mathrm{g}$ TNP-KLH (Biosearch Technologies), $25 \mu \mathrm{g}$ TNP-Ficoll (Biosearch Technologies), or $50 \mu \mathrm{g}$ OVA emulsified in Alhydrogel (Invivogen) and boosted with antigen on day 14. Blood was collected from mice via retroorbital bleed at the time of immunization and 21 days after the initial immunization. Mice were treated with TUDCA (MilliporeSigma) at a concentration of 500 $\mathrm{mg} / \mathrm{kg}$ or saline control i.p daily throughout the immunization.

Cytotoxicity assays. T cell and NK cell cytotoxicity assays were performed as described in the Supplemental Methods.

Cohousing with pet store mice. Pet store mice were purchased from a retailer and 1 pet store mouse was cohoused in the same cage with four 8- to 12-week-old WT or Copg1 ${ }^{K 652 E}$ mice in an ABSL2 facility. Mice were treated with TUDCA (MilliporeSigma) at a concentration of 500 $\mathrm{mg} / \mathrm{kg}$ or saline control i.p daily throughout the cohousing experiment. The repertoire of microbes of pet store, cohoused, and SPF mice were determined using the PRIA array (Charles River Laboratories) on feces collected upon experimental termination.

Study approval. Human studies were approved by the Boston Children's Hospital Institutional Review Board. Written informed consent was obtained from the patients' parents or guardians. All mouse studies were approved and performed in accordance with Boston Children's Hospital Institutional Animal Research and Care Committee.

Statistics. All data are presented as mean \pm SEM. Statistical significance for single comparisons was calculated using the 2-sided Student's $t$ test, 2-way ANOVA, Mann-Whitney $U$ test, or Holm-Šídák test to control for multiple comparisons (GraphPad). Statistical significance for multiple comparisons was computed as specified in the figure legends. A $P$ value of less than 0.05 was considered significant.

\section{Author contributions}

WB, CDP, SYP, KS, JGW, ZTP, MJM, MB, SAS, JJ, SBC, FJ, JSY, and CR performed the experiments. SURN and SAT collected and analyzed the clinical data. WB, VWH, TCW, SRN, JSO, JC, MT, and RSG analyzed the data and wrote the manuscript.

\section{Acknowledgments}

The authors thank R.V. Farese Jr. and B. Croker for useful discussions, F.S. Alkuraya and H.E. Shamseldin for performing and analyzing the WES, and E. Janssen for the autoantibody screen. This work is supported by NIH grants K08AI116979-04 (to JC), R01AI139633 (to RSG), R37GM058615 (to VWH), R01GM097194 (to TCW), and R01AI120989 (to JSO), the Perkin Fund, and 5T32AI007512-33 (to SBC).

Address correspondence to: Raif S. Geha, Children's Hospital Boston, 1 Blackfan Circle, Karp 10, Boston, Massachusetts 02115, USA. Phone:1.617.919.2482; Email: raif.geha@childrens.harvard.edu.

MJM's present address is: Faculty of Medicine, American University of Beirut, Beirut, Lebanon.
1. Feige MJ, et al. How antibodies fold. Trends Biochem Sci. 2010;35(4):189-198.

2. Pelham HR. The retention signal for soluble proteins of the endoplasmic reticulum. Trends Biochem Sci. 1990;15(12):483-486.

3. Orci L, et al. Bidirectional transport by distinct populations of COPI-coated vesicles. Cell. 1997;90(2):335-349.

4. Hsu VW, et al. The evolving understanding of COPI vesicle formation. Nat Rev Mol Cell Biol. 2009;10(5):360-364.

5. Cosson P, et al. New COP1-binding motifs involved in ER retrieval. EMBO J. 1998;17(23):6863-6870.

6. Eugster A, et al. The alpha- and beta'-COP WD40 domains mediate cargo-selective interactions with distinct di-lysine motifs. Mol Biol Cell. 2004;15(3):1011-1023. 
7. Letourneur F, et al. Coatomer is essential for retrieval of dilysine-tagged proteins to the endoplasmic reticulum. Cell.1994;79(7):1199-1207.

8. Michelsen K, et al. Novel cargo-binding site in the beta and delta subunits of coatomer. JCell Biol. 2007;179(2):209-217.

9. Jackson LP, et al. Molecular basis for recognition of dilysine trafficking motifs by COPI. Dev Cell. 2012;23(6):1255-1262.

10. Ma W, Goldberg J. Rules for the recognition of dilysine retrieval motifs by coatomer. EMBO J. 2013;32(7):926-937.

11. Ron D, Walter P. Signal integration in the endoplasmic reticulum unfolded protein response. Nat Rev Mol Cell Biol. 2007;8(7):519-529.

12. Bertolotti A, et al. Dynamic interaction of BiP and ER stress transducers in the unfolded-protein response. Nat Cell Biol. 2000;2(6):326-332.

13. Shen J, et al. Stable binding of ATF6 to BiP in the endoplasmic reticulum stress response. Mol Cell Biol. 2005;25(3):921-932.

14. Shamu CE, Walter P. Oligomerization and phosphorylation of the Ire1p kinase during intracellular signaling from the endoplasmic reticulum to the nucleus. EMBO J.1996;15(12):3028-3039.

15. Haze K, et al. Mammalian transcription factor ATF6 is synthesized as a transmembrane protein and activated by proteolysis in response to endoplasmic reticulum stress. Mol Biol Cell. 1999;10(11):3787-3799.

16. Yoshida $\mathrm{H}$, et al. XBP1 mRNA is induced by ATF6 and spliced by IRE1 in response to ER stress to produce a highly active transcription factor. Cell. 2001;107(7):881-891.

17. Shaffer AL, et al. XBP1, downstream of Blimp-1, expands the secretory apparatus and other organelles, and increases protein synthesis in plasma cell differentiation. Immunity. 2004;21(1):81-93.

18. Reimold AM, et al. Plasma cell differentiation requires the transcription factor XBP-1. Nature. 2001;412(6844):300-307.

19. Harding HP, et al. Regulated translation initiation controls stress-induced gene expression in mammalian cells. Mol Cell. 2000;6(5):1099-1108.

20. Matsumoto M, et al. Ectopic expression of CHOP (GADD153) induces apoptosis in M1 myeloblastic leukemia cells. FEBS Lett. 1996;395(2-3):143-147.

21. Yamaguchi H, Wang HG. CHOP is involved in endoplasmic reticulum stress-induced apoptosis by enhancing DR5 expression in human carcinoma cells. JBiol Chem. 2004;279(44):45495-45502.

22. Pino SC, et al. CHOP mediates endoplasmic reticulum stress-induced apoptosis in Gimap5-deficient T cells. PLoS One. 2009;4(5):e5468.

23. Masciarelli S, et al. CHOP-independent apoptosis and pathway-selective induction of the UPR in developing plasma cells. Mol Immunol. 2010;47(6):1356-1365.

24. Ma Y, et al. Plasma cell differentiation initiates a limited ER stress response by specifically suppressing the PERK-dependent branch of the unfolded protein response. Cell Stress Chaperones. 2010;15(3):281-293.

25. Kurtulus S, et al. Bcl-2 allows effector and memory $\mathrm{CD}^{+} \mathrm{T}$ cells to tolerate higher expression of Bim. JImmunol. 2011;186(10):5729-5737.

26. Watson PJ, et al. Gamma-COP appendage domain - structure and function. Traffic. 2004;5(2):79-88.

27. Yang JS, et al. A role for phosphatidic acid in COPI vesicle fission yields insights into Golgi maintenance. Nat Cell Biol. 2008;10(10):1146-1153.

28. Yang JS, et al. A role for BARS at the fission step of COPI vesicle formation from Golgi membrane. EMBO J. 2005;24(23):4133-4143.

29. Yang JS, et al. COPI acts in both vesicular and tubular transport. Nat Cell Biol. 2011;13(8):996-1003.

30. Cole NB, et al. Retrograde transport of Golgi-localized proteins to the ER. J Cell Biol. 1998;140(1):1-15.

31. Yang JS, et al. ARFGAP1 promotes the formation of COPI vesicles, suggesting function as a component of the coat. J Cell Biol. 2002;159(1):69-78.

32. Cosson P, Letourneur F. Coatomer interaction with di-lysine endoplasmic reticulum retention motifs. Science. 1994;263(5153):1629-1631.

33. Lee SY, et al. ARFGAP1 plays a central role in coupling COPI cargo sorting with vesicle formation. J Cell Biol. 2005;168(2):281-290.

34. Lee AH, et al. XBP-1 regulates a subset of endoplasmic reticulum resident chaperone genes in the unfolded protein response. Mol Cell Biol. 2003;23(21):7448-7459.

35. Wiest DL, et al. Membrane biogenesis during B cell differentiation: most endoplasmic reticulum proteins are expressed coordinately. JCell Biol. 1990;110(5):1501-1511.

36. Moon HB, et al. Regulation of IgG1 and IgE synthesis by interleukin 4 in mouse B cells. Scand J Immunol. 1989;30(3):355-361.

37. Xu Z, et al. Immunoglobulin class-switch DNA recombination: induction, targeting and beyond. Nat Rev Immunol. 2012;12(7):517-531.

38. Xie Q, et al. Effect of tauroursodeoxycholic acid on endoplasmic reticulum stress-in- duced caspase-12 activation. Hepatology. 2002;36(3):592-601.

39. Sun JC, Lanier LL. NK cell development, homeostasis and function: parallels with $\mathrm{CD}^{+} \mathrm{T}$ cells. Nat Rev Immunol. 2011;11(10):645-657.

40. Beura LK, et al. Normalizing the environment recapitulates adult human immune traits in laboratory mice. Nature. 2016;532(7600):512-516.

41. Reese TA, et al. Sequential infection with common pathogens promotes human-like immune gene expression and altered vaccine response. Cell Host Microbe. 2016;19(5):713-719.

42. Masopust D, et al. Of mice, dirty mice, and men: using mice to understand human immunology. J Immunol. 2017;199(2):383-388.

43. Takeda AJ, et al. Human PI3K $\gamma$ deficiency and its microbiota-dependent mouse model reveal immunodeficiency and tissue immunopathology. Nat Commun. 2019;10(1):4364.

44. Moelleken J, et al. Differential localization of coatomer complex isoforms within the Golgi apparatus. Proc Natl Acad Sci U S A. 2007;104(11):4425-4430.

45. Blanc $\mathrm{P}$, et al. Mature IgM-expressing plasma cells sense antigen and develop competence for cytokine production upon antigenic challenge. Nat Commun. 2016;7:13600.

46. Zhang K, Kaufman RJ. From endoplasmic-reticulum stress to the inflammatory response. Nature. 2008;454(7203):455-462.

47. Cao SS, et al. Endoplasmic reticulum stress interacts with inflammation in human diseases. JCell Physiol. 2016;231(2):288-294.

48. Watkin LB, et al. COPA mutations impair ER-Golgi transport and cause hereditary autoimmune-mediated lung disease and arthritis. Nat Genet. 2015;47(6):654-660.

49. Izumi K, et al. ARCN1 mutations cause a recognizable craniofacial syndrome due to COPI-mediated transport defects. Am J Hum Genet. 2016;99(2):451-459.

50. DiStasio A, et al. Copb2 is essential for embryogenesis and hypomorphic mutations cause human microcephaly. Hum Mol Genet. 2017;26(24):4836-4848.

51. Jabara HH, et al. A missense mutation in TFRC, encoding transferrin receptor 1 , causes combined immunodeficiency. Nat Genet. 2016;48(1):74-78.

52. Alkuraya FS. Autozygome decoded. Genet Med. 2010;12(12):765-771.

53. Park SY, et al. Coordinated regulation of bidirectional COPI transport at the Golgi by CDC42. Nature. 2015;521(7553):529-532. 